\title{
Modeling steel fiber reinforced concrete: numerical immersed boundary approach and a phenomenological mesomodel for concrete-fiber interaction
}

\author{
A. $\operatorname{Pros}^{1}$, P. Diez ${ }^{1, *, \dagger}$ and C. Molins ${ }^{2}$ \\ ${ }^{1}$ Laboratori de Càlcul Numèric, Departament de Matemàtica Aplicada III, Universitat Politècnica de Catalunya, \\ BarcelonaTech, Campus Nord UPC, 08034 Barcelona, Spain \\ ${ }^{2}$ Departament d'Enginyeria de la Construcció, Universitat Politècnica de Catalunya, BarcelonaTech, Campus Nord \\ UPC, 08034 Barcelona, Spain
}

\begin{abstract}
SUMMARY
Steel fiber reinforced concrete (SFRC) allows overcoming brittleness and weakness under tension, the main drawbacks of plain concrete. The influence of the fibers on the behavior of SFRC depends on their shape, length, slenderness, and also on their orientation and distribution into the plain concrete. The goal of this paper is to develop an ad hoc numerical strategy to account for the contribution of the fibers in the simulation of the mechanical response of SFRC. In the model presented, the individual fibers immersed in the concrete bulk are accounted for in their actual location and orientation. The selected approach is based on the ideas introduced in the immersed boundary (IB) methods. These methods were developed to account for 1D (or 2D) solids immersed in 2D (or 3D) fluids. Here, the concrete bulk is playing the role of the fluid and the cloud of steel fibers is acting as the immerse boundary (that is, a 1D structure in a $2 \mathrm{D}$ or 3D continuous). Thus, the philosophy of the IB methodology is used to couple the behavior of the two systems, the concrete bulk and fiber cloud, precluding the need of matching finite element meshes. Note that, considering the different size scales and the intricate geometry of the fiber cloud, the conformal matching of the meshes would be a restriction resulting in a practically unaffordable mesh.

In the proposed approach, the meshes of the concrete bulk and fiber cloud are independent, and the models are coupled imposing displacement compatibility and equilibrium of the two systems. In the applications presented here, the concrete bulk is modeled using a standard nonlinear damage model. The constitutive model for the fibers is designed to account for the complex interaction between fibers and concrete. The fiber models are based on the previous investigations describing the concrete-fiber interaction and its dependence on the factors identified to be relevant: shape of the fiber (straight or hooked) and angle between the fiber and crack plane.
\end{abstract}

KEY WORDS: plain concrete; steel fiber reinforced concrete; numerical modeling; constitutive equations; continuous models; discontinuous models; immersed boundary method; fiber-concrete interaction; pullout tests; direct tension tests

\section{INTRODUCTION}

The most common and used techniques for overcoming the main drawbacks of plain concrete (its brittleness and weakness under tension) are reinforced and prestressed concrete. Another alternative, presented in the 1970s, is steel fiber reinforced concrete (SFRC), a technique which consists of adding steel fibers into the plain concrete matrix, as another component of the concrete mixture.

Steel fiber reinforced concrete has a large range of applications in civil engineering (bridges, pipes, airport runways, tunnel linings, pavements,...). For characterizing the behavior of SFRC,

*Correspondence to: P. Diez, Modul C2, Campus Nord UPC, 08034 Barcelona, Spain.

†E-mail: pedro.diez@upc.edu 
fibers shape, length, and slenderness must be taken into account in addition to the steel and concrete matrix properties. Moreover, it is also necessary to take into account the orientation and distribution of the fibers inside the concrete bulk. The fracture energy and the residual strength increase because of the presence of steel fibers into plain concrete, particularly, in tension.

Several test methods may be used to determine, direct or indirectly, the tensile behavior of SFRC. Bending tests of prismatic beam specimens are the most used to characterize the post-cracking response of SFRC. These can be either based on three-point tests [1,2] or four-point tests [3]. Other methods have also been used for the material characterization; some of them are indirect tension tests-splitting test [4] and Barcelona test ([5], UNE 83515) — and direct tension tests [6]. Barcelona test is the extension of the double punch test [5] to the post-cracking response of SFRC. In spite of being the uniaxial tensile test, the more direct approach to characterize SFRC in tension, presents difficulties inherent to their execution which hugely limit their use and current application [7], and the number of results available.

Once SFRC is characterized experimentally, a numerical modeling tool is necessary to describe the response of this material in more complex setuns.

A number of authors have recently introduced new models for SFRC. For instance, a numerical model considering an elasto-plastic material model for plain concrete with an exponential softening law turning into a linear softening law once all the fibers are activated is presented in [8]. The crack propagation in SFRC considering a fracture mechanics approach is simulated in [9].

On the other hand, in [10], the compressive damage zone model (which is defined for plain concrete [11]) is adapted for SFRC, adding extra parameters corresponding to steel fibers. The same author studies the tensile behavior of SFRC considering a numerical model presented by [12]. Also in [12], pullout tests are used to characterize fibers behavior keeping conformal the concrete and
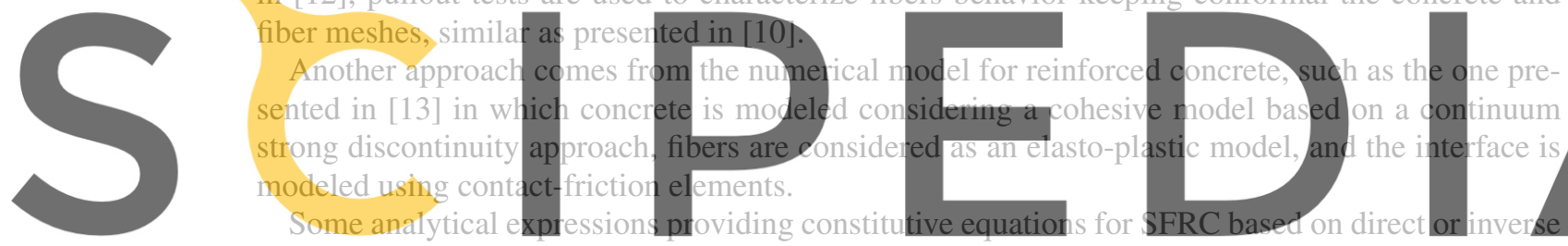

approaches giving macroscopic approximations are presented in [14]. In such constitutive models,

the homogenized models do not account for the actual distribution of the fibers.

Most of the numerical methods proposed in the past have a common characteristic: concrete buik and fiber cloud meshes are conformal (with geometrical matching). Therefore, building up a conformal mesh for the whole system is cumbersome and computationally expensive. For pullout tests, considering only one fiber, conformal meshes are useful and relatively easy to generate. However, if several fibers are considered, these models are severely limited because of their computational cost.

There are, however, previous attempts of using nonconformal meshes. These strategies were first introduced for standard reinforced concrete and recently, also for SFRC. For instance, [15] and [16] introduce the idea of embedded reinforcement approach to deal with structures including curved walls with regular reinforcements. A similar idea is presented in [17] for concrete structures discretized with structured meshes and curved or draped reinforcements. Reference [18] introduces a nonconformal approach to model Fiber Reinforced Concrete (FRC) based on the partition-of-theunity finite element method. In [19], the nonlinear behavior of FRC is presented following the same ideas introduced in [18]. Moreover, in [20], only a background mesh (corresponding to the concrete matrix) is considered, and the fibers (not discretized explicitly to ensure computational efficiency of the model) are represented by interaction forces.

In the present work, an alternative for modeling numerically SFRC is presented, introduced in [21]. The goal is to avoid conformal meshes and homogenized models. In the current proposal, concrete and fiber meshes are independent, nonconformal, and the actual geometry of all the fibers is defined inside the concrete mesh. Moreover, although concrete and fiber models interact, they are defined independently. The main idea considered herein for coupling the two models is based on the immersed boundary (IB) methods [22-24], which were introduced for a fluid with a solid immersed 
in it (here, concrete is thought as the fluid, and the fibers are like the solid structure immersed in the fluid). Then, displacement compatibility between the two models is imposed. In order to describe the whole debonding process between the fibers and plain concrete, a mesomodel for steel fibers is presented, which is translated into the constitutive equations for fibers. These constitutive equations depend on the angle between the fiber and normal direction of the failure pattern. They also depend on the shape of the fiber (straight or hooked). The model for plain concrete is defined independently, being typically a nonlinear model.

The remainder of this paper is structured as follows. First, in Section 2, the problem statement of SFRC is presented. In Section 3, the discrete approach of the problem is described. Section 4 presents the models considered for each material: (1) a nonlinear mesomodel for steel fibers accounting for the whole behavior between plain concrete and steel fibers and (2) nonlinear models for plain concrete. Then, in Section 5, two numerical examples of the proposed model are presented: a pullout test (considering different fiber orientations) and the direct tension test considering both plain concrete and SFRC (with straight and hooked fibers). The paper is closed with some concluding remarks and persnective future work.

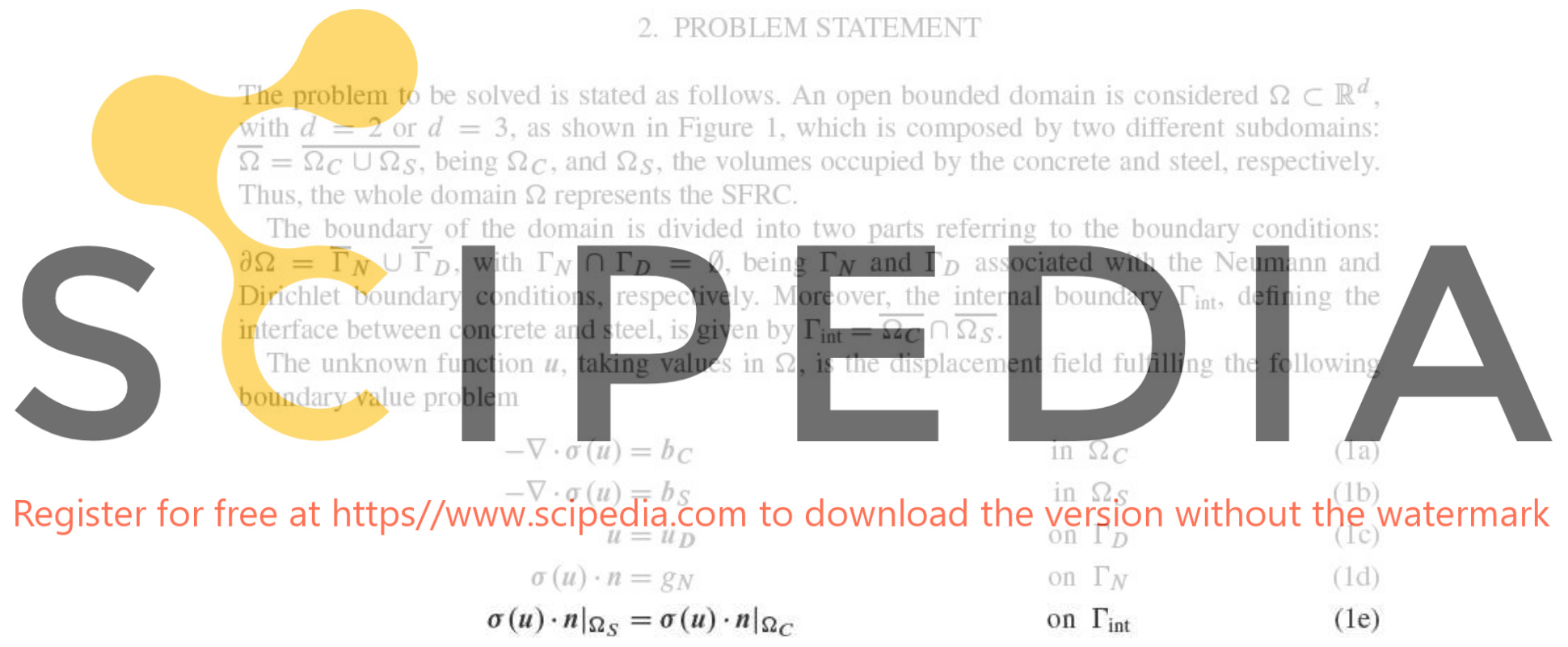

where $\boldsymbol{b}_{C}$ and $\boldsymbol{b}_{S}$ stand for the body loads in the concrete and steel, and $\boldsymbol{u}_{\boldsymbol{D}}$ and $g_{N}$ are the prescribed displacements and tractions on $\Gamma_{D}$ and $\Gamma_{N}$.

For solving the current problem, different options could be considered. As pointed out in the introduction, one possibility is defining conformal meshes for the two domains $\Omega_{C}$ and $\Omega_{S}$ with different material models. Another option consists in considering a homogenized model for the complete

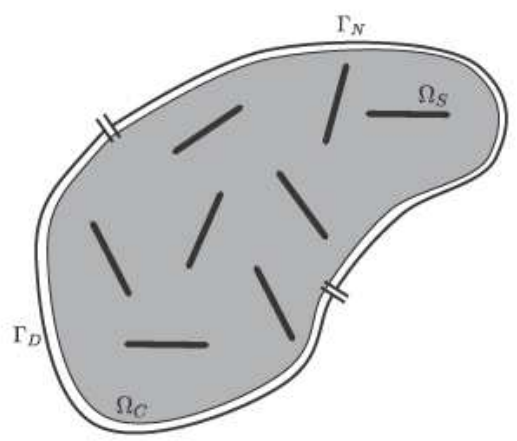

Figure 1. General domain. 
domain. In the present paper, a third option is presented avoiding conformal meshes (too expensive and not affordable for large number of steel fibers) and homogenized models (not accounting for the actual geometry of the fibers).

The proposed approach is based on the IB methods [22-24] which were introduced for solving problems considering a solid structure immersed in a fluid. The main idea of these methods is to neglect the space occupied by the solid structure. The fluid is considered to occupy the whole domain, and the velocities of both solid and fluid are made compatible in the coinciding points. Then, the effect of the solid in the fluid is accounted for by adding an interaction force. The two systems (fluid and solid structure) are considered separately and compatibility is enforced by adding the corresponding interaction forces. The discretization of the problem is therefore simplified because the mesh of the whole domain (the fluid) may be very simple (eventually a cartesian mesh), and the mesh for the solid body does not require to be conformal with it. The models corresponding to the fluid and solid are defined independently.

Here, the same approach is used, taking the plain concrete the role of the fluid and the fiber cloud the role of the solid structure. Therefore, the problem to be solved is redefined. First, the geometrical conception is adapted to the new scheme. It is assumed that the geometrical support of the fibers is a 1D manifold (with measure zero in $\mathbb{R}^{d}$ ). Thus, $\Omega_{S}$ is replaced by an ideal version $\widetilde{\Omega_{S}}$, see Figure 2 . The two subdomains $\Omega_{C}$ and $\Omega_{S}$ are therefore replaced by $\Omega$ and $\widetilde{\Omega_{S}}$, respectively. Note that the fiber eloud domain, $\widetilde{\Omega_{S}}$, is defined overlapping the new concrete domain, $\Omega$, that is, for each fiber point, there is another point in the concrete background with the same coordinates.

Secondly, the problem statement is reformulated by adapting the equilibrium equations (1) to this new geometry. The unknown displacement field $u$ takes values in every point $x \in \Omega$. The com-
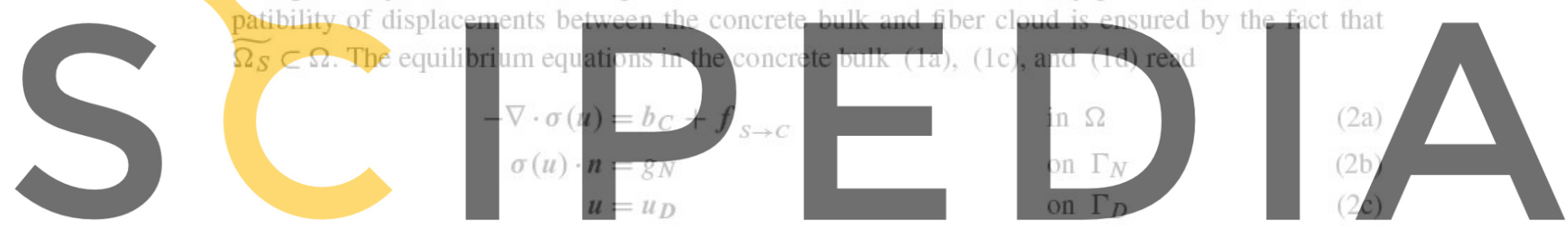

being $f$, the interaction force accounting for the effect of the fibers in the concrete bulk Register for free at htfps/dwww,scipedia.com to download the version without the watermark

The interaction force $f_{S \rightarrow C}$ is defined taking into account the equilibrium equation in the fibers, (1b). An arc length $s$ is introduced for each fiber using the parametrization $X(s)$, as illustrated in Figure 3. The displacement along the fiber is given by the displacement field $u$ taking values in the concrete bulk; the restriction of $\boldsymbol{u}$ to $\widetilde{\Omega_{S}}$ is denoted by $\boldsymbol{u}_{S}$.

The expression for $f_{S \rightarrow C}$ at a point $x \in \widetilde{\Omega_{S}} \subset \Omega$ is given by

$$
f_{S \rightarrow C}(x)=\int_{\widetilde{\Omega_{S}}} f(s) \delta_{0}(x-X(s)) d s .
$$

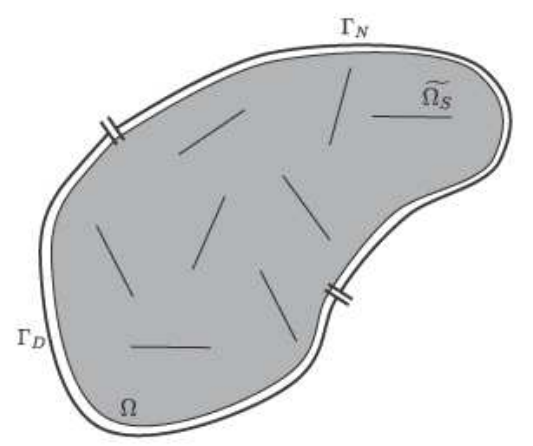

Figure 2. Ideal domain. 

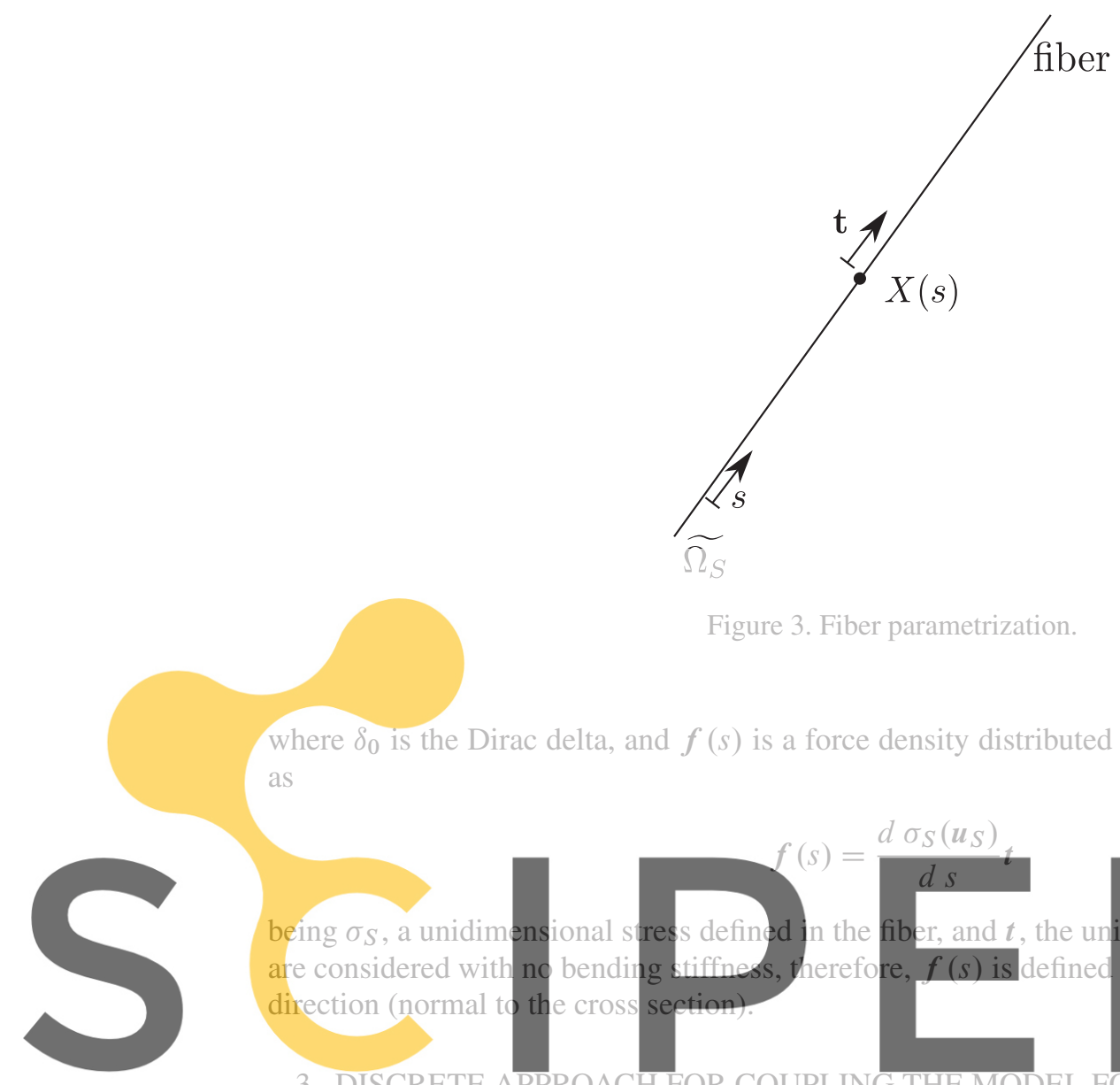

where $\delta_{0}$ is the Dirac delta, and $f(s)$ is a force density distributed along the fiber line and defined as

\section{Register for free at https//www.scipedia.com to download the version without the watermark}

The problem is discretized defining independently one mesh for the concrete bulk and one mesh for the fiber cloud. The mesh for the concrete bulk is kept simple while preserving the geometrical features of the sample, for instance, the notch, see Figure 4. The discretization of the fiber cloud is a series of straight bar elements (in the examples included in this work each fiber is discretized with five elements). No conformity or geometrical matching is enforced between the discretizations of the concrete bulk and fiber cloud. For a given discretization, the corresponding unknowns of the discrete problem are the nodal values of the displacements in the concrete bulk and in the steel fiber cloud. These nodal vectors are denoted by $u_{c}$ and $u_{s}$, respectively, and they are a priori independent. The displacement compatibility must be enforced specifically. The discrete form of the equilibrium equations (2) (equilibrium in the concrete bulk), is expressed in terms of nodal force vectors and reads

$$
F_{c}^{\mathrm{int}}=F_{c}^{\mathrm{ext}}+F_{s \rightarrow c},
$$

where the nodal vector $F_{c}^{\text {ext }}$ is the discrete version of the external force vector and accounts for the effect of $\boldsymbol{b}_{C}$ and $g_{N}, F_{S \rightarrow c}$ stands for the discrete form of $\boldsymbol{f}_{S \rightarrow C}$ and $F_{c}^{\text {int }}$ corresponds to the internal forces, that is, the discrete vector version of $-\nabla \cdot \sigma(\boldsymbol{u})$.

A similar expression holds for the equilibrium in the fiber cloud

$$
F_{s}^{\mathrm{int}}=F_{s}^{\mathrm{ext}}+F_{c \rightarrow s}
$$

where the first terms are analogous to the previous equation, and $F_{c \rightarrow s}$ is an interaction force from the concrete bulk into the steel fibers. 


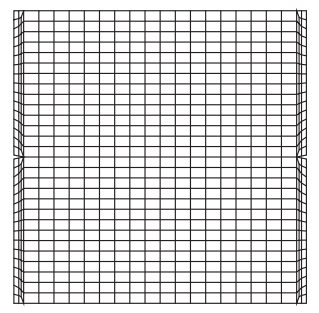

Concrete bulk
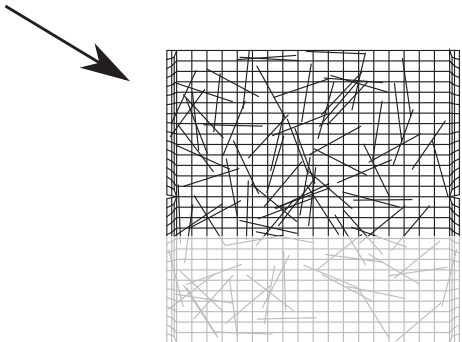

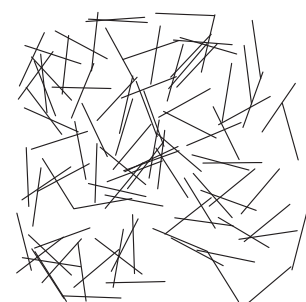

Fiber cloud

SFRC
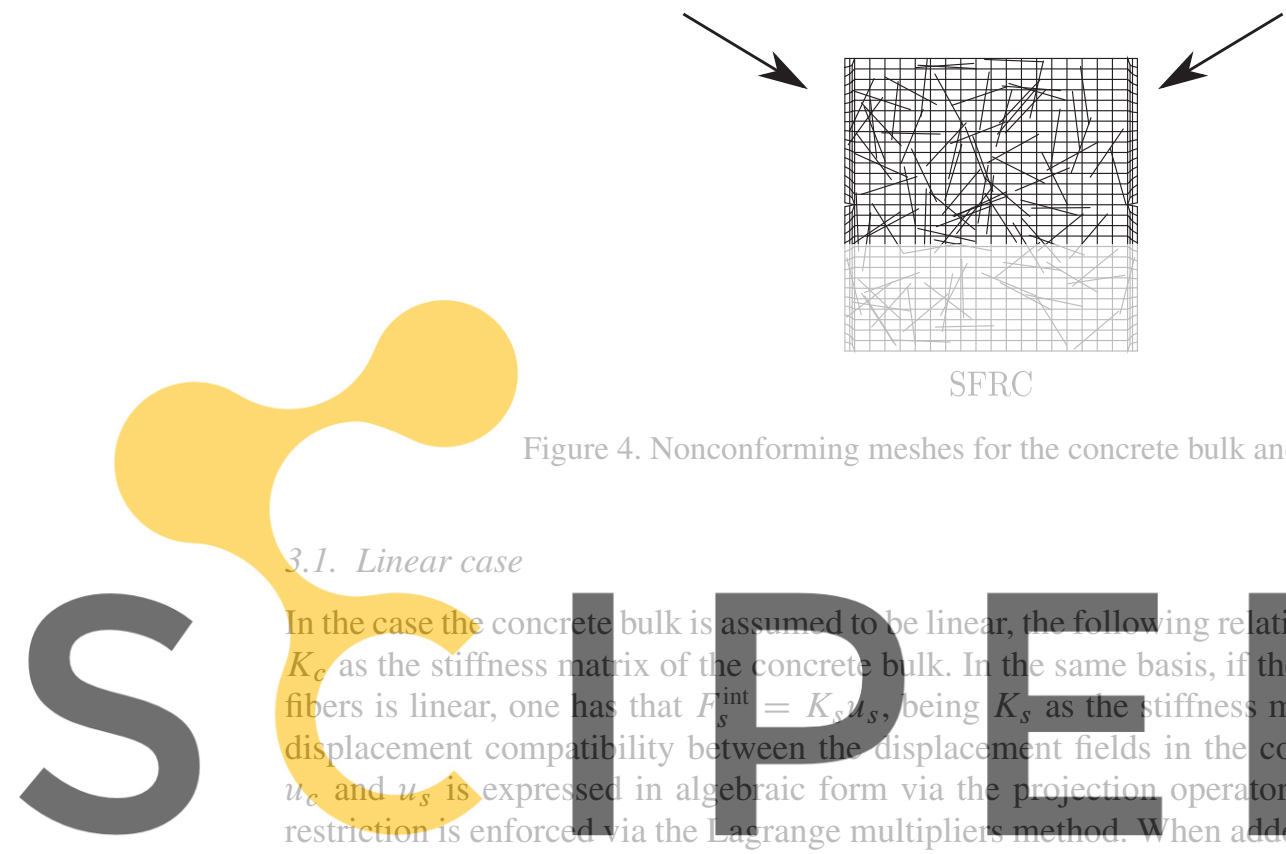

\section{Figl}

Figure 4. Nonconforming meshes for the concrete bulk and fiber cloud.

equations (5) and (6), the following system yields

Register for free at https//www.scipedia.com to download the version without the watermark

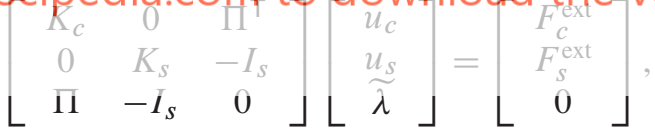

Note that the number of rows of the matrix $\Pi$ corresponds to the number of DOF of the fibers, and its number of columns is the number of DOF of the concrete bulk.

In order to identify the discrete interaction forces, $F_{s \rightarrow c}$ and $F_{c \rightarrow s}$, the previous system is rewritten as

$$
\left\{\begin{array}{rlrl}
K_{c} u_{c} & =F_{c}^{\mathrm{ext}}-\Pi^{\top} \widetilde{\lambda} & \text { concrete equilibrium } \\
K_{s} u_{s} & =F_{s}^{\mathrm{ext}}+\widetilde{\lambda} & \text { fiber cloud equilibrium } \\
\Pi u_{c}=u_{s} & \text { displacement compatibility }
\end{array}\right.
$$

that is, the interaction force from the fibers to the concrete is $F_{s \rightarrow c}\left(u_{s}\right)=-\Pi^{\top} \widetilde{\lambda}$, and the interaction force from the concrete to the fibers is precisely the Lagrange multiplier, $F_{c \rightarrow s}\left(u_{c}\right)=\widetilde{\lambda}$.

In the remainder of the paper and according with the hypothesis of neglecting the volume of the steel fibers, the external forces on the fibers are also assumed to be zero, $F_{s}^{\text {ext }}=0$.

\section{Remark 1}

Essential boundary conditions in the concrete bulk. In SFRC, Dirichlet boundary conditions are imposed only in the concrete bulk. The general form of a linear restriction is $A u_{c}^{*}=u_{D}^{*}, A$ being a rectangular matrix and $u_{D}^{*}$ the vector of prescribed values. Considering Lagrange multipliers for 
imposing these boundary conditions, the concrete stiffness $\left(K_{c}\right)$, displacement $\left(u_{c}\right)$, and the external forces $\left(F_{c}^{\text {ext }}\right)$ are defined as follows:

$$
K_{c}=\left[\begin{array}{cc}
K_{c}^{*} & A^{\top} \\
A & 0
\end{array}\right], \quad u_{c}=\left[\begin{array}{c}
u_{c}^{*} \\
\lambda^{*}
\end{array}\right] \text { and } F_{c}^{\mathrm{ext}}=\left[\begin{array}{c}
F_{c}^{\mathrm{ext} *} \\
u_{D}^{*}
\end{array}\right]
$$

being $K_{c}^{*}$ and $u_{c}^{*}$, the structural concrete stiffness and displacement, respectively, without the Lagrange multipliers. Vector $\lambda^{*}$ is the Lagrange multiplier, and $F_{c}^{\text {ext* }}$ represents the external load applied in the concrete bulk.

Therefore, the system to be solved for the problem considering the Lagrange multipliers method without accounting for the fibers interaction would be

$$
K_{c} u_{c}=F_{c}^{\mathrm{ext}} \text {. }
$$

Thus, this allows writing in a unified manner the loading term, both if displacements or forces are prescribed.

\subsection{Nonlinear case}

In a realistic case, fibers and concrete are modeled as nonlinear materials. Thus, the general form of the discrete nonlinear system to be solved reads
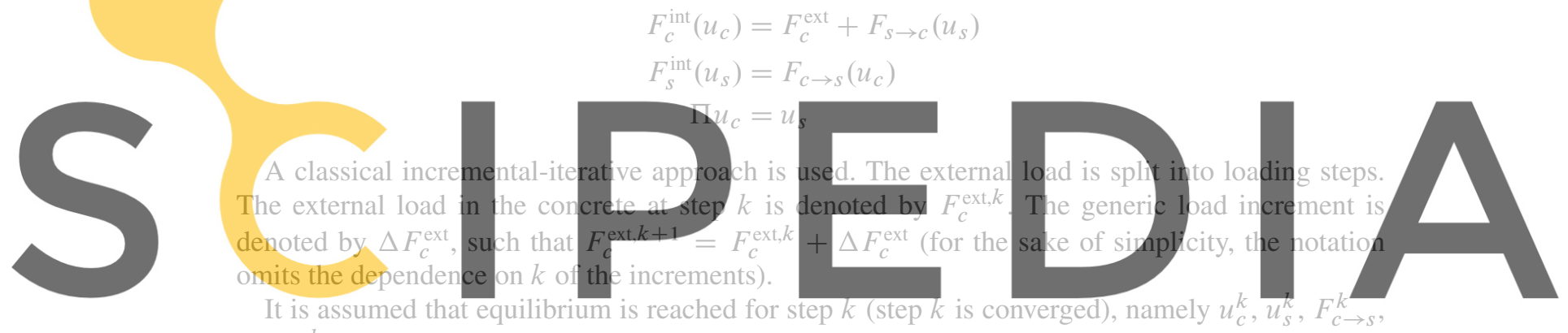

$$
\begin{aligned}
F_{c}^{\text {int }}\left(u_{c}^{k}\right) & =F_{c}^{\text {ext }, k}+F_{c \rightarrow s}^{k} \\
F_{c}^{\text {int }}\left(u_{c}^{k}\right) & =F_{s \rightarrow c}^{k} \\
\Pi u_{c}^{k} & =u_{s}^{k}
\end{aligned}
$$

Then, we seek the solution at the next step $k+1$. The problem reads, given the solution at step $k$ and $\Delta F_{c}^{\text {ext }}$, find $u_{c}^{k+1}, u_{s}^{k+1}, F_{c \rightarrow s}^{k+1}$, and $F_{s \rightarrow c}^{k+1}$ satisfying

$$
\begin{aligned}
F_{c}^{\mathrm{int}}\left(u_{c}^{k+1}\right) & =F_{c}^{\mathrm{ext}, k+1}+F_{c \rightarrow s}^{k+1}=F_{c}^{\mathrm{ext}, k}+\Delta F_{c}^{\mathrm{ext}}+F_{c \rightarrow s}^{k+1} \\
F_{s}^{\mathrm{int}}\left(u_{s}^{k+1}\right) & =F_{s \rightarrow c}^{k+1} \\
\Pi u_{c}^{k+1} & =u_{s}^{k+1}
\end{aligned}
$$

In order to solve the incremental nonlinear problem, an iterative method is used. Iterations are required for finding $\Delta u_{c}$ and $\Delta u_{s}$ such as

$$
\begin{aligned}
& u_{c}^{k+1}=u_{c}^{k}+\Delta u_{c} \\
& u_{s}^{k+1}=u_{s}^{k}+\Delta u_{s}
\end{aligned}
$$

Therefore, assuming the approximation

$$
\begin{aligned}
& F_{c}^{\mathrm{int}}\left(u_{c}^{k+1}\right) \approx F_{c}^{\mathrm{int}}\left(u_{c}^{k}\right)+K_{c} \Delta u_{c} \\
& F_{s}^{\mathrm{int}}\left(u_{c}^{k+1}\right) \approx F_{s}^{\mathrm{int}}\left(u_{s}^{k}\right)+K_{s} \Delta u_{s},
\end{aligned}
$$


the initial trial increment (iteration $i=0$ ) aims at determining the approximations $\delta u_{c, 0}$ and $\delta u_{s, 0}$ to $\Delta u_{c}$ and $\Delta u_{s}$. The first iteration is then computed by solving the following system of equations:

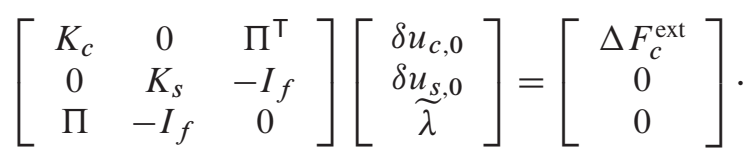

being $\widetilde{\lambda}$ as the corresponding Lagrange multiplier.

For the next iterations, $i=1,2,3 \ldots$, the displacement increments are assumed to have the form $\Delta u_{c} \approx \sum_{j=0}^{i} \delta u_{c, j}$ and $\Delta u_{s} \approx \sum_{j=0}^{i} \delta u_{s, j}$.

Because the approximation to $u_{c}^{k+1}$ and $u_{s}^{k+1}$ at iteration $i-1$ is available, the residual is computed as

$$
\begin{aligned}
& r_{c, i-1}=F_{c}^{\mathrm{ext}, k+1}-F_{c}^{\mathrm{int}}\left(u_{c}^{k+1}\right)-\Pi^{\top} \widetilde{\lambda} \\
& r_{s, i-1}=F_{s}^{\text {int }}\left(u_{s}^{k+1}\right)-\tilde{\lambda}
\end{aligned}
$$

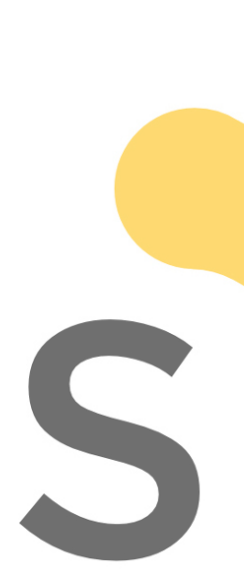

The next iteration $i$ is obtained solving
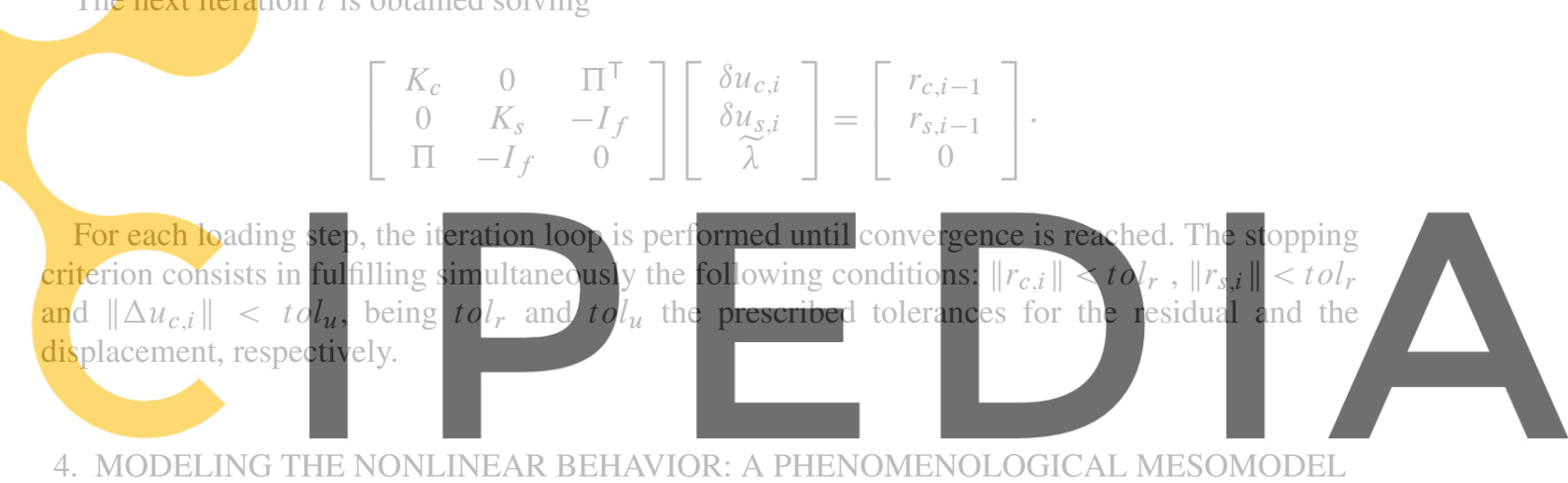

Register for free at https/fwww.scipedia.com to download the version without the watermark

steel fiber cloud independently. This section is devoted to describe the models used for both mate-

rials. A classical nonlocal damage model is proposed for concrete. The steel fiber cloud is modeled using an elasto-plastic mesomodel accounting for the interaction between plain concrete and steel fibers.

\subsection{Plain concrete}

Concrete is a brittle material which can be modeled considering both continuous and discontinuous models. In [25], two different alternatives are considered for plain concrete. On one hand, a continuous model is known as Mazars damage model. In this case, the model is considered nonlocal in order to avoid mesh dependence. The failure pattern is obtained, and the value of the maximum vertical load (which is the variable chosen for validation of numerical models) is satisfactory. On the other hand, a discontinuous model is considered; the failure pattern is known a priori (through the experimental and numerical results from considering the continuous model) and it is modeled considering joint elements (allowing both sliding and separation), whereas the rest of the specimen is elastic. Although it is necessary to know the fracture pattern before the simulation, the results are also close to the experimental ones.

Therefore, these alternatives can be considered for modeling plain concrete, as well as any other nonlinear model available and suitable for plain concrete.

Another useful damage model is the one presented in [26] with less material parameters and easier to control than the Mazars damage model. For the examples in the present work, this damage model is considered. The general formulation of this damage model is introduced in [26] and [27]. 
In the latter, the author shows that the model is regularized because it involves an internal length parameter.

\subsection{Steel fibers and concrete-fiber interaction}

The model considered for steel fibers accounts for the whole process of slipping and debonding of the fiber into plain concrete, and precisely, allows capturing the whole behavior between the fibers and plain concrete bulk. Therefore, an elasto-plastic angle dependent model with softening is adopted for the steel fibers. The interaction is modeled with the monolithic model imposing displacement compatibility described in the previous sections of the present paper (based in the IBM). The concept of monolithic strategy is used here by opposition to the strategy devised in [21] in which the linear system of equations is solved using a staggered scheme corresponding to a block Gauss-Seidel method.

An alternative approach would be considering steel fibers being modeled with an elasto-plastic model and the interaction between concrete and fibers defined describing the whole process (slipping, folding, debonding, etc.). Although the steel fiber model would be easy, the interaction is more complex to perform.

The mesomodel for the steel fibers is expected to account for the whole interaction process between concrete and fibers and to characterize the behavior of the steel fibers. This aims at describing the effect of different phenomena, resulting in a global behavior at the mesoscale. The mesomodel is assumed to include in a single constitutive relation the effects of the nonlinear behavior of the steel, the slipping, folding, debonding, and also the sliding of the fiber with respect to the concrete. Thus, the constitutive equations of steel fibers are deduced from experimental results and
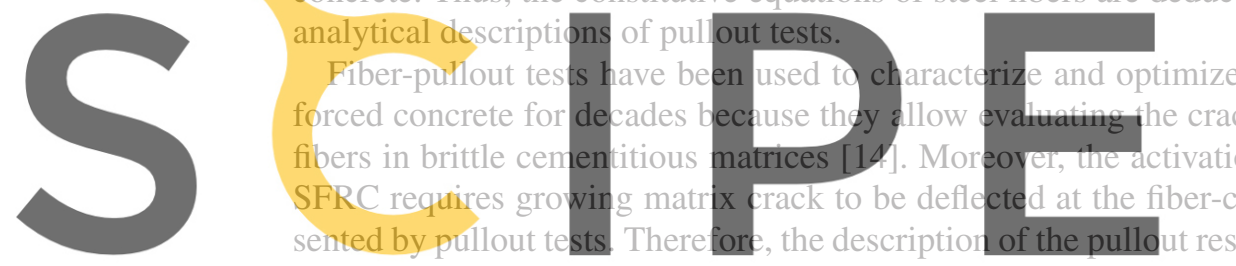

sented by pullout tests. Therefore, the description of the pullout res

phenomena with which crack bridging of SFRC can be explained. However, pullout and bond-slip

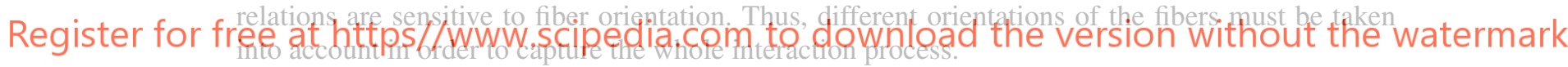

Pullout tests describes all the phenomena of the SFRC not only for straight fibers (fiber debonding, matrix spalling, frictional sliding, and fiber removal), but also for hooked fibers (which are the same for the case of straight fibers but with plastic deformations, magnifying the matrix spalling effects).

Pullout tests consist of a plain concrete specimen with only one steel fiber immersed on it, which is pulled out. In Figure 5, the scheme of a pullout test is presented. Fortunately, there is a recent analytical phenomenological description of pullout tests available based on experimental results [14]. These analytical expressions depend on the angle between the fiber and load direction $(\phi)$, represented in Figure 5, and on the shape of the fiber, which can be straight or hooked (as displayed in Figure 6). The whole expressions are described in Appendix A.

For the case of considering straight fibers, the pullout results presented in [28] are reproduced in Figure 7. Different results are presented considering different orientations of the fibers (with reference to the load direction).

In the present work, the outputs from the pullout tests, crack width, and pullout load $(w, P)$ are translated into the constitutive equations for the steel fibers $(\varepsilon, \sigma)$ following the expressions for a given discretization of the steel fibers

$$
\begin{aligned}
& P \rightarrow \sigma=\frac{P}{A_{\text {fiber }} \cdot \cos \phi} \\
& w \rightarrow \varepsilon=\left\{\begin{array}{ccc}
\frac{w \cdot \cos \phi}{L} & \text { if } & w \leqslant w_{1} \\
\frac{w_{1} \cdot \cos \phi}{L}+\frac{\left(w-w_{1}\right) \cdot \cos \phi}{L_{\text {elem }}} & \text { if } & w \geqslant w_{1}
\end{array}\right.
\end{aligned}
$$




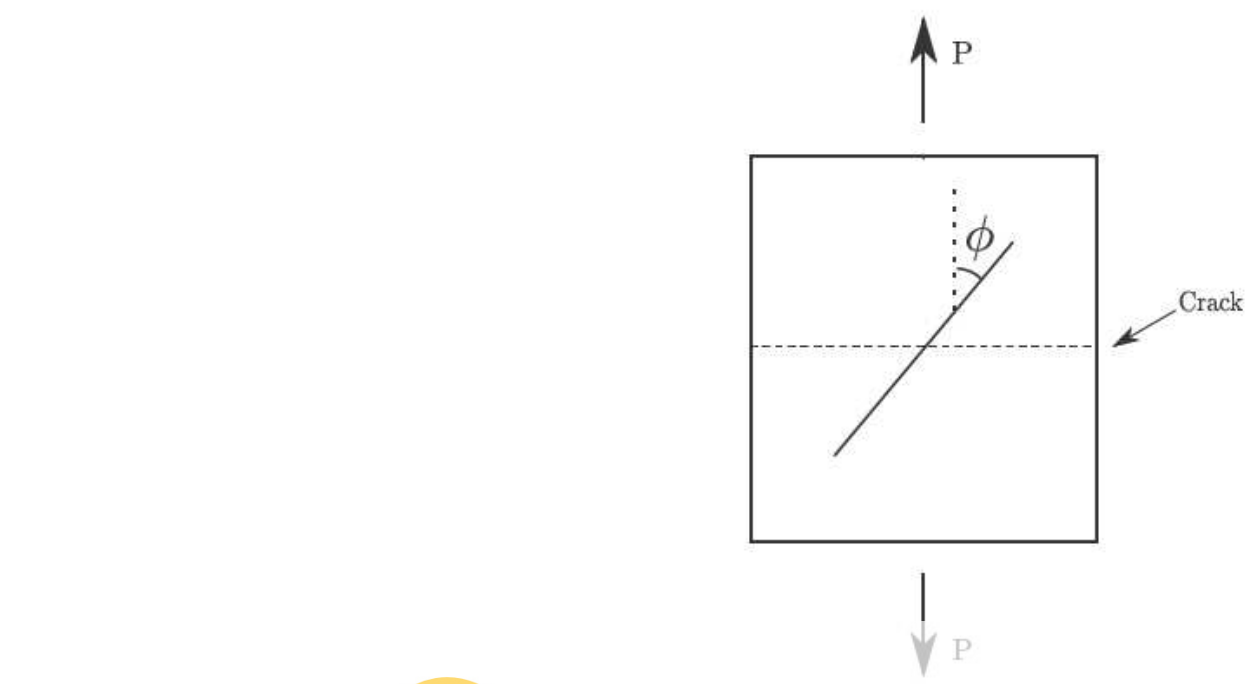

Figure 5. Pullout test scheme with the angle $(\phi)$ between the fiber and loading direction.
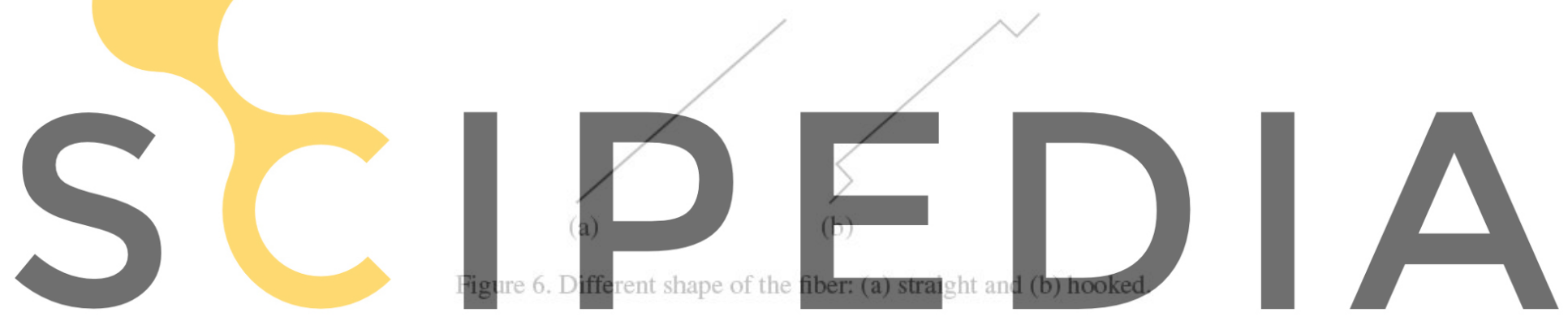

Register for free at https//www.scipedia.com to download the version without the watermark
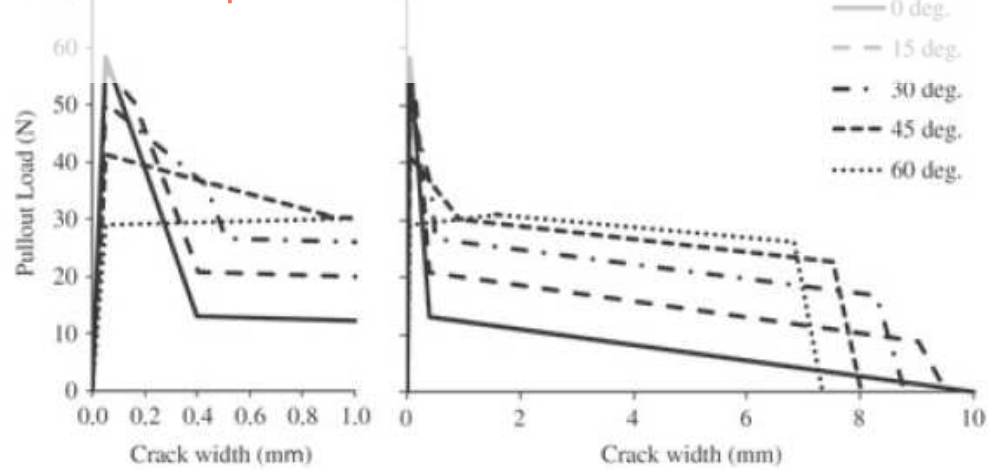

Figure 7. Pullout test results considering straight fibers for different values of $\phi$, image from [28].

with $A_{\text {fiber }}$ and $L$ standing for the area and length of the fiber, respectively, and $L_{\text {elem }}$ being the element size of the fiber (for the given discretization). It is considered that although the fiber has not plastified $\left(w \leqslant w_{1}\right)$, the whole geometry of the fiber has the same behavior. However, once the fiber has plastified $\left(w \geqslant w_{1}\right)$, the deformation of the fiber is supposed to be concentred only in one element of the fiber.

$(w, P)$ are defined in the axis of the pullout test, as presented in Figure 8, and the constitutive equations of the fibers $(\varepsilon, \sigma)$ must be defined in the axis of the fiber. Therefore, it is necessary to 


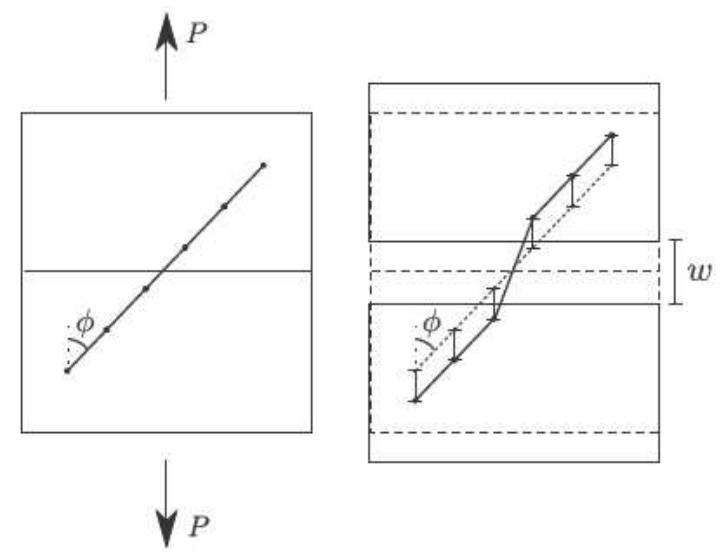

Figure 8. $w$ and $P$ before and after the cracking.

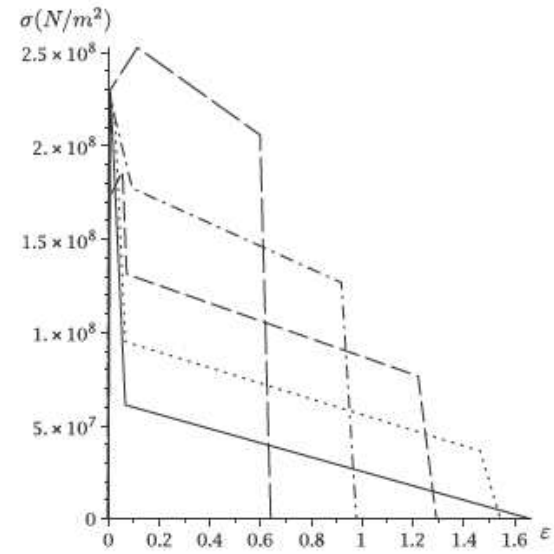

(a)

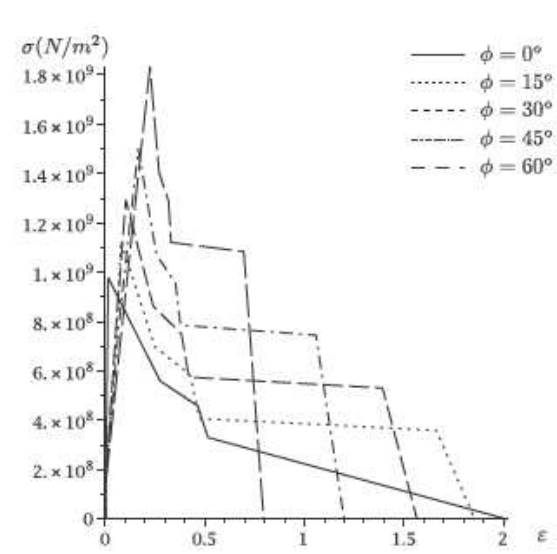

(b)

Figure 9. Constitutive equations for steel fibers with different orientation considering both straight (a) and hooked (b) shapes.

project $(w, P)$ into the fiber $\left(w_{F}, P_{F}\right)$, being $P_{F}=P \cos \phi$ the tension of the fiber and $w_{F}=\frac{w}{\cos \phi}$ the fiber elongation associated with the crack.

In general, the angle is computed between the fiber and normal direction of the fracture pattern. However, for the pullout tests, the load direction is perpendicular to the failure pattern, therefore, the normal direction coincides with the load direction.

In the common situations, the volumetric proportion of steel fibers into plain concrete is $\leqslant 1 \%$. In this case, the behavior of the fiber inside concrete is the same as if it was isolated, and it is not necessary taking into the account the fiber-fiber interaction. However, if the volumetric proportion of fibers is larger than $1 \%$, this assumption may be unrealistic. Accounting for the fiber-fiber interaction is out of the scope of this paper.

Therefore, for each fiber immersed in the concrete bulk, a different constitutive equation is considered depending on its shape (straight or hooked) and on the angle between the considered fiber and normal direction of the failure pattern. In Figure 9, different constitutive equations are presented for different angles and considering both straight and hooked fibers. These equations are obtained through analytical expressions of the pullout tests and defined in the fiber axis.

It is observed that the presented constitutive equations are angle-dependent with softening and at the final stages of the deformation, the stresses $(\sigma)$ tend to zero. 


\section{EXAMPLES}

After describing the numerical approach for modeling plain concrete, two numerical examples are presented. On one hand, two pullout tests considering different orientations of the fibers are showed, and on the other hand, a direct tension test is simulated. Both cases are academic examples in two dimensions under the condition of plane stress.

In all the examples presented in the present work, plain concrete is modeled with the damage model explained in [26]. For all cases such as plain concrete and SFRC, the material parameters considered are the same (Table I).

\subsection{Pullout test}

Herein, two pullout tests are reproduced. The size of the fibers considered in both cases is presented in Table II, and the concrete specimen size is $25 \mathrm{~mm} \times 12.5 \mathrm{~mm}$.

First, as represented in Figure 10(a), a straight single fiber with no inclination with respect to the load direction is considered immersed on the plain concrete. The steel fiber is fixed (in the bottom part of the concrete specimen, which is not included in the model) and the top part of the concrete bulk is pulled up considering prescribed displacements at the top of the specimen. The nodes of the fiber embedded in the lower part are blocked, assuming that all the deformation is concentrated in the rest of the fiber. Plain concrete is simulated considering a damage model. The constitutive equation of the steel fiber is taken from Figure 9(a) and Appendix A for the case of straight fibers and $\phi=0^{\circ}$. Moreover, in Figure 10(b), the results are displayed in a load-displacement curve.

Table I. Material parameters for the damage model for plain concrete.

\begin{tabular}{ll}
\hline Young modulus & $E=30 \cdot 10^{9} \mathrm{~Pa}$ \\
Poisson coefficient & $\mu=0$ \\
Fracture energy & $\sigma_{u}=10 \mathrm{~N}$ \\
Element size & $l_{e}=0.5 \mathrm{~mm}$ \\
Tensile strength & $f_{t}=3.5 \cdot 10^{6} \mathrm{~Pa}$ \\
\hline
\end{tabular}

Table II. Fiber dimensions for the pullout tests.

\begin{tabular}{lcc}
\hline Length & Embedded length & Diameter \\
\hline $30 \mathrm{~mm}$ & $10 \mathrm{~mm}$ & $0.5 \mathrm{~mm}$ \\
\hline
\end{tabular}

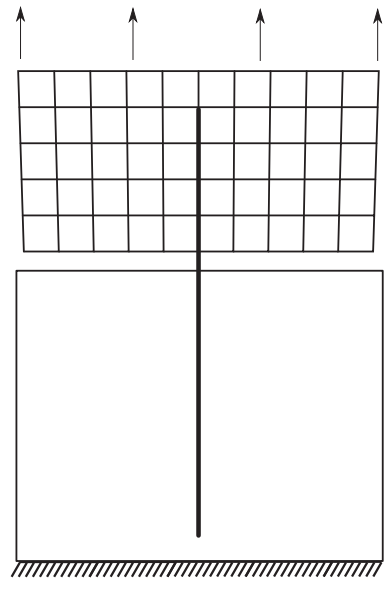

(a)

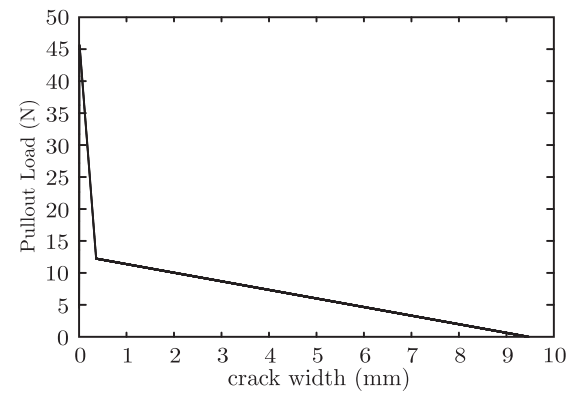

(b)

Figure 10. Pullout test considering $\phi=0^{\circ}$. 
Qualitatively, the behavior of the numerical model is in good agreement with the corresponding results obtained experimentally [28] and to the curves presented in Figure 7. In order to obtain a quantitative matching of the model, the concrete model has to be further validated.

After considering a straight fiber with no inclination, another case is simulated, the pullout test considering an inclined straight fiber with $\phi=30^{\circ}$ immersed in the plain concrete. Again, displacements are prescribed at the top of the plain concrete specimen and the fiber is fixed. Herein, the model for the plain concrete is the same than in the previous case, the damage model, but the constitutive equation of the fiber is different, based on the information from Figure 9(a) and Appendix A. In Figure 11(a), the scheme of the example is presented, and in Figure 11(b), the obtained results are shown in a load-displacement curve which is the expected one [28].

In both examples of pullout tests, it is observed that the obtained curve $(w, P)$ is in good agreement with the results obtained experimentally and to the analytical curves presented in [28] corresponding to each case (depending on the orientation of the fiber), as expected. That is because of the fact that the constitutive equation of each fiber is deduced form pullout results depending on the angle.

\subsection{Direct tension test}

The direct tension test is simulated considering three different cases: (1) only plain concrete (Figure 12(a)); (2) SFRC with straight fibers (Figure 12(b)); and (3) SFRC with hooked fibers (Figure 12(c)). The direct tension test consists in a rectangular specimen made of plain concrete or SFRC fixed at the bottom and under a direct load pulling at the top (shown in Figure 12), precisely, prescribed displacements are considered at the top of the concrete specimen. The size of the specimen of plain concrete is $75 \mathrm{~mm} \times 75 \mathrm{~mm}$ in all the examples of the direct tension loading presented. The simulation is carried out considering plane stress and the considered thickness of the specimen is $75 \mathrm{~mm}$.

In order to avoid damage dispersion and to ensure having the crack pattern placed in the same place for all the cases, a notch is performed in the three meshes.

For the case of SFRC, the steel fibers are distributed and orientated randomly into the plain concrete bulk. Both the location of one end-point, $p_{i}$, and the angle of the fiber with respect to the horizontal $\alpha$ are assumed to be random variables with uniform probability distributions. That means that for rectangular domains, each coordinate of $p_{i}$ ranges in a real interval and $\alpha$ ranges in $[0, \pi[$ (three random variables in $2 \mathrm{D}$ that would turn out to be five, three coordinates, and two angles in 3D). Because the length of the fibers, $L$, is given as problem data, once $p_{i}$ and $\alpha$ are generated, the

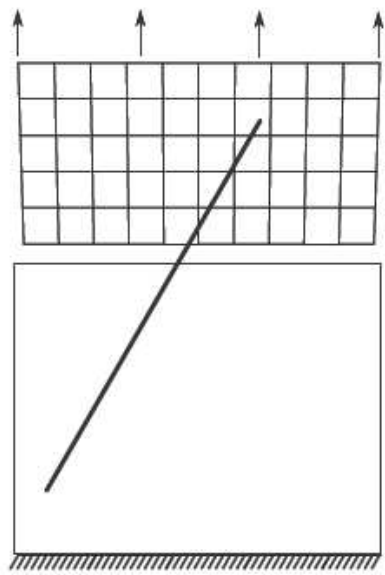

(a)

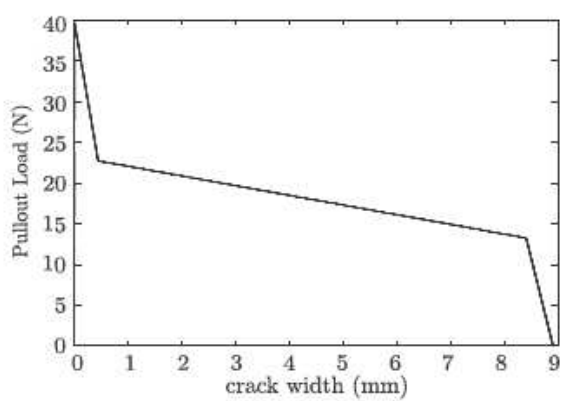

(b)

Figure 11. Pullout test considering $\phi=30^{\circ}$. 


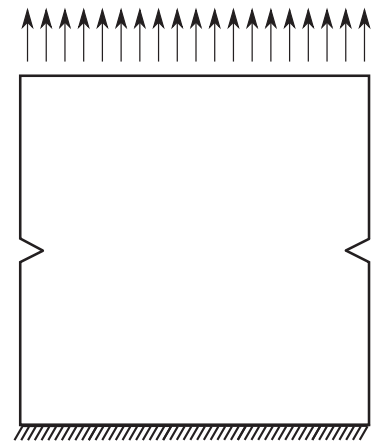

(a)

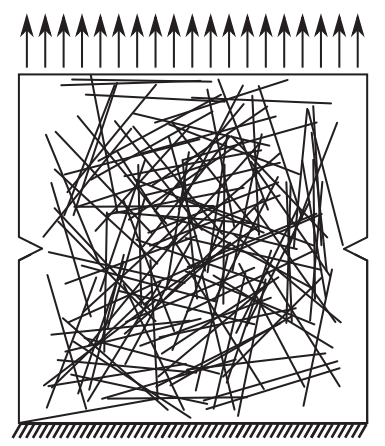

(b)

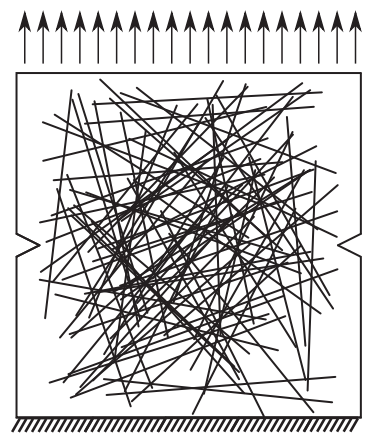

(c)

Figure 12. Direct tension test considering (a) plain concrete, (b) steel fiber reinforced concrete with straight fibers, and (c) steel fiber reinforced concrete with hooked fibers.

Table III. Fiber dimensions.

\begin{tabular}{llcc}
\hline Shape & Length & Diameter & Number of fibers \\
\hline Straight & $30 \mathrm{~mm}$ & $0.5 \mathrm{~mm}$ & 140 \\
Hooked & $50 \mathrm{~mm}$ & $0.5 \mathrm{~mm}$ & 84 \\
\hline
\end{tabular}

other end-point of the fiber is readily computed by doing

$$
p_{f}=p_{i}+L(\cos \alpha, \sin \alpha) .
$$

If the end-point $p_{f}$ lies outside of the concrete domain, the fiber generated is discarded.

In Table III, the dimensions of the fibers (based on [14]) for each case are displayed.

For the two cases of SFRC, as the length of the fibers is not the same for straight fibers than for hooked fibers, different quantities of fibers are considered into plain concrete. In order to compare under the same conditions, the same volumetric substitution amount of steel fibers into plain concrete are considered: $0.23 \%$.

For the steel fibers, the constitutive equations considered are based on Figure 9 and Appendix A. The corresponding angle between the fiber and load direction (which is perpendicular to the failure zone, and thus, coincides with the normal direction of the failure pattern) is computed, and then the corresponding constitutive equation is defined considering the equations and the input data presented in Appendix A.

In Figure 13, the obtained results from the simulation of the direct tension test are presented; three load-displacement curves (one for each case: plain concrete, SFRC with straight fibers, and SFRC with hooked fibers) in which one can observe (1) the increase of the fracture energy and (2) the appearance of the residual strength due to the presence of the steel fibers into plain concrete. Moreover, it is observed that the appeared residual strength is higher for the SFRC with hooked fibers than for the SFRC with straight fibers. Therefore, the effects of the fibers are captured, and moreover, it is observed that hooked fibers influence more than straight fibers into the plain concrete. For a better analysis of the results, a zoom near to the load peak is done and presented in Figure 14.

In order to study the behavior in all the cases, three sampling times (pseudo-time in the quasistatic loading process) are selected, $T_{1}, T_{2}$, and $T_{3}$, as represented in Figure 13. The initial time step is represented by $T_{0}$. On one hand, Figures 15-17 show the deformed mesh of plain concrete in this three different time steps and in the initial step, for the three different materials: plain concrete, SFRC considering straight fibers, and SFRC with hooked fibers. The influence of the fibers is observed in these deformed meshes.

Note that the deformed meshes for both straight and hooked fibers are practically equal (Figures 16 and 17). The differences between the two types of fibers appear in the load-displacement 


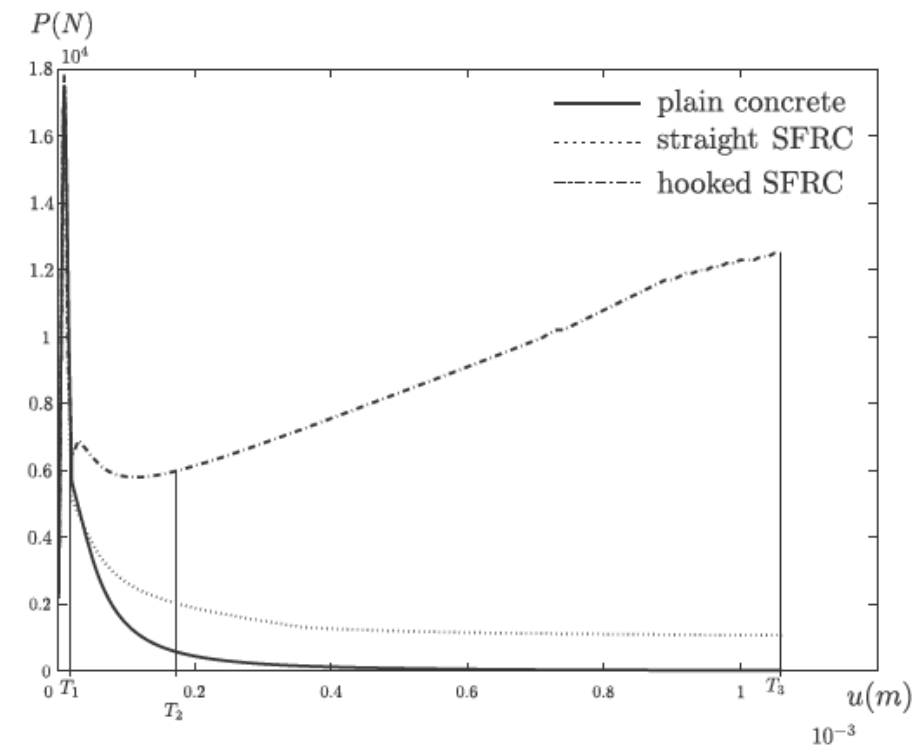

Figure 13. Direct tension test results considering plain concrete, steel fiber reinforced concrete with straight fibers, and steel fiber reinforced concrete with hooked fibers.

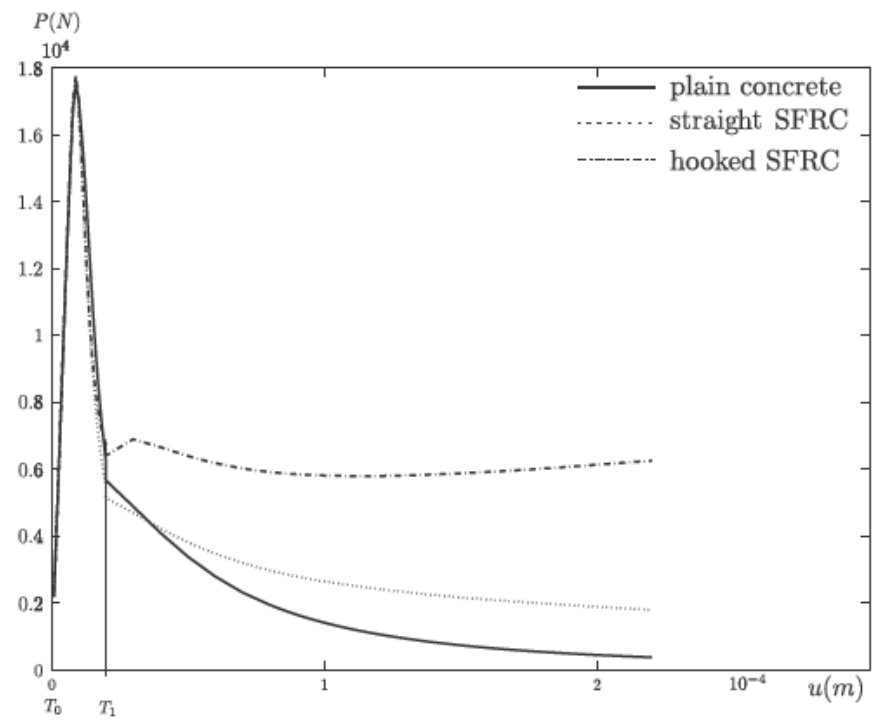

Figure 14. Zoom of the direct tension test results considering plain concrete, steel fiber reinforced concrete with straight fibers, and steel fiber reinforced concrete with hooked fibers.

curves (Figures 13 and 14). The tests are displacement driven, and therefore, the displacement fields (and hence the deformed meshes) are similar.

On the other hand, Figures 18 and 19 show the fibers that are not in the elastic range (plastified) in the different time steps for the case of SFRC considering straight and hooked fibers, respectively. The number of the plastified fibers is presented in brackets for each case. It is observed that the plastified fibers are the ones that cross the fracture pattern, and the number of plastified fibers increases with the time. Moreover, there are more plastified hooked fibers than straight fibers because of the fact that hooked fibers are longer, and therefore, more hooked fibers cross the fracture pattern.

For the whole comprehension of the behavior, three straight and three hooked fibers are studied in Figures 20 and 21 considering the three time steps located into the constitutive equation of each 


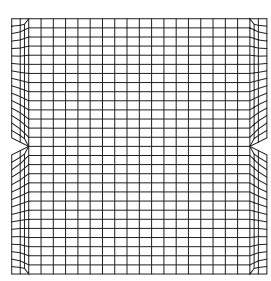

$T_{0}$

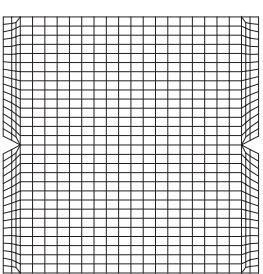

$T_{1}$

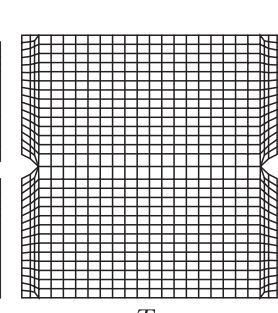

$T_{2}$

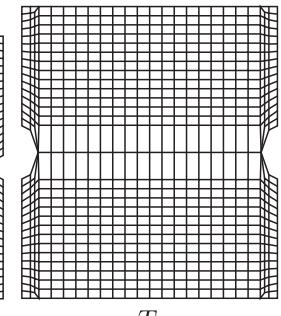

$T_{3}$

Figure 15. Deformed meshes for plain concrete $($ amplified $\times 10)$.

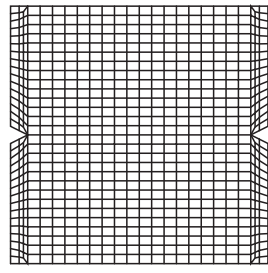

$T_{0}$

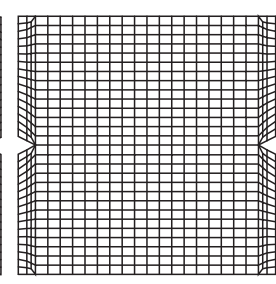

$T_{1}$

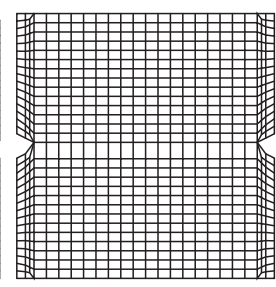

$T_{2}$

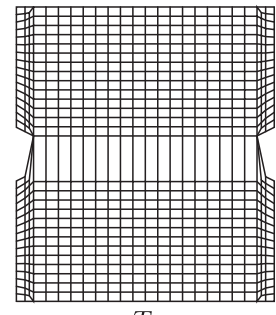

$T_{3}$

Figure 16. Deformed meshes for straight steel fiber reinforced concrete (amplified $\times 10)$.

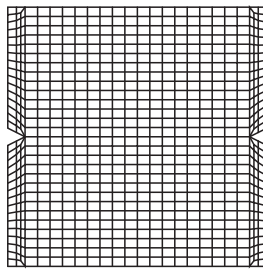

$T_{0}$

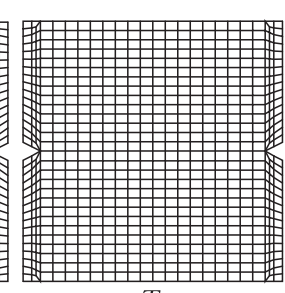

$T_{1}$

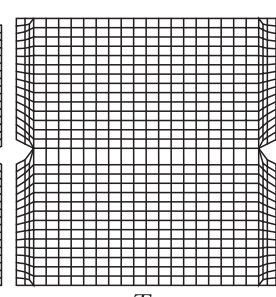

$T_{2}$

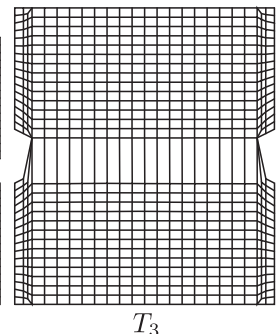

$T_{3}$

Figure 17. Deformed meshes for hooked steel fiber reinforced concrete (amplified $\times 10)$.

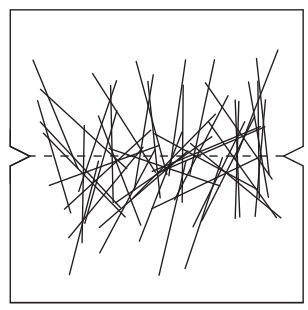

$T_{1}(47)$

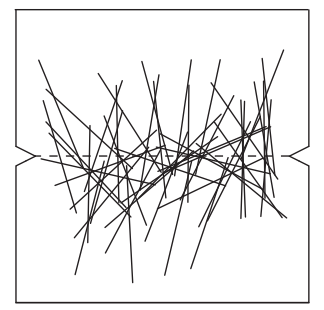

$T_{2}(50)$

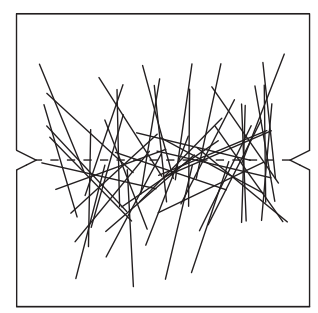

$T_{3}(51)$

Figure 18. Straight fibers that have plastified in $T_{1}, T_{2}$, and $T_{3}$ with the number of plastified fibers in brackets.

different fiber for both cases. All the studied fibers cross the fracture pattern, therefore, are not in the elastic range, as observed.

\section{CONCLUDING REMARKS}

A new numerical strategy is presented to numerically simulate the SFRC. The main features of the proposed approach are the following:

(i) The meshes of the concrete bulk and fiber cloud are defined independently (nonconformal). 

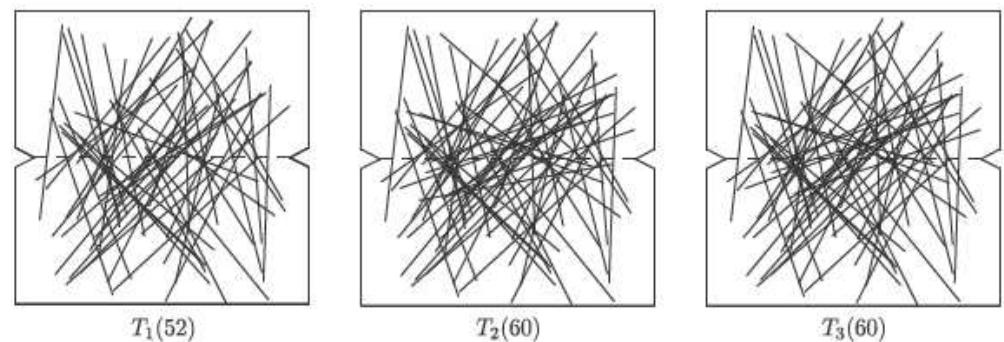

Figure 19. Hooked fibers that have plastified in $T_{1}, T_{2}$, and $T_{3}$ with the number of plastified fibers in brackets.
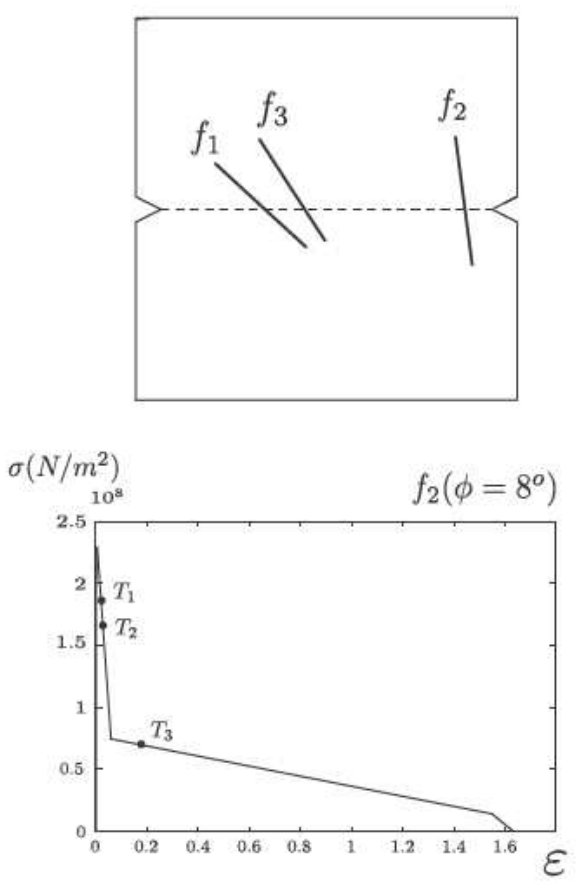
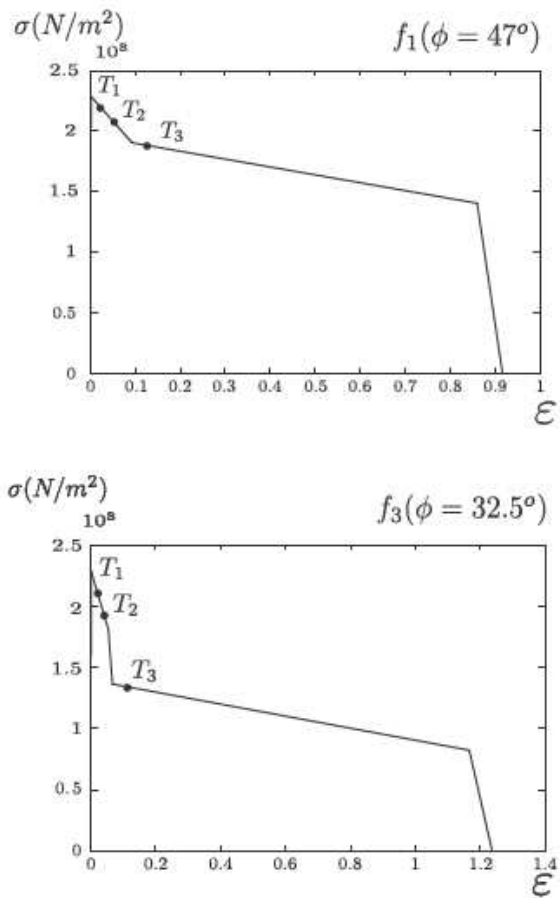

Figure 20. Straight fibers: behavior of three straight fibers $\left(f_{1}, f_{2}\right.$, and $\left.f_{3}\right)$ crossing the crack with the constitutive equation of each fiber.

(ii) The material models of the concrete bulk and fiber cloud (which accounts also for the fiber-concrete interaction) are defined separately.

(iii) A phenomenological mesomodel is developed to characterize the constitutive equations for the steel fibers, accounting for the interaction with the concrete bulk.

(iv) Coupling of the two systems (concrete and fibers) is based on the IB methods, imposing displacement compatibility and equilibrium.

The mesomodel is defined from the phenomenological analytical expressions describing the behavior of pullout tests. Thus, the constitutive equation for each steel fiber depends on (1) the angle between the fiber and normal direction of the failure pattern (for the pullout test, the load direction coincides to the normal direction) and (2) the shape of the fiber. Further research has to be carried out to properly define the angle between each fiber and the crack pattern provided by the numerical model of the plain concrete bulk (the damage distribution). This is especially relevant in $3 \mathrm{D}$ cases.

The main advantage of this strategy is the possibility of using the actual number of fibers, with their location and orientation. In the examples, randomly distributed fiber clouds have been used. In 

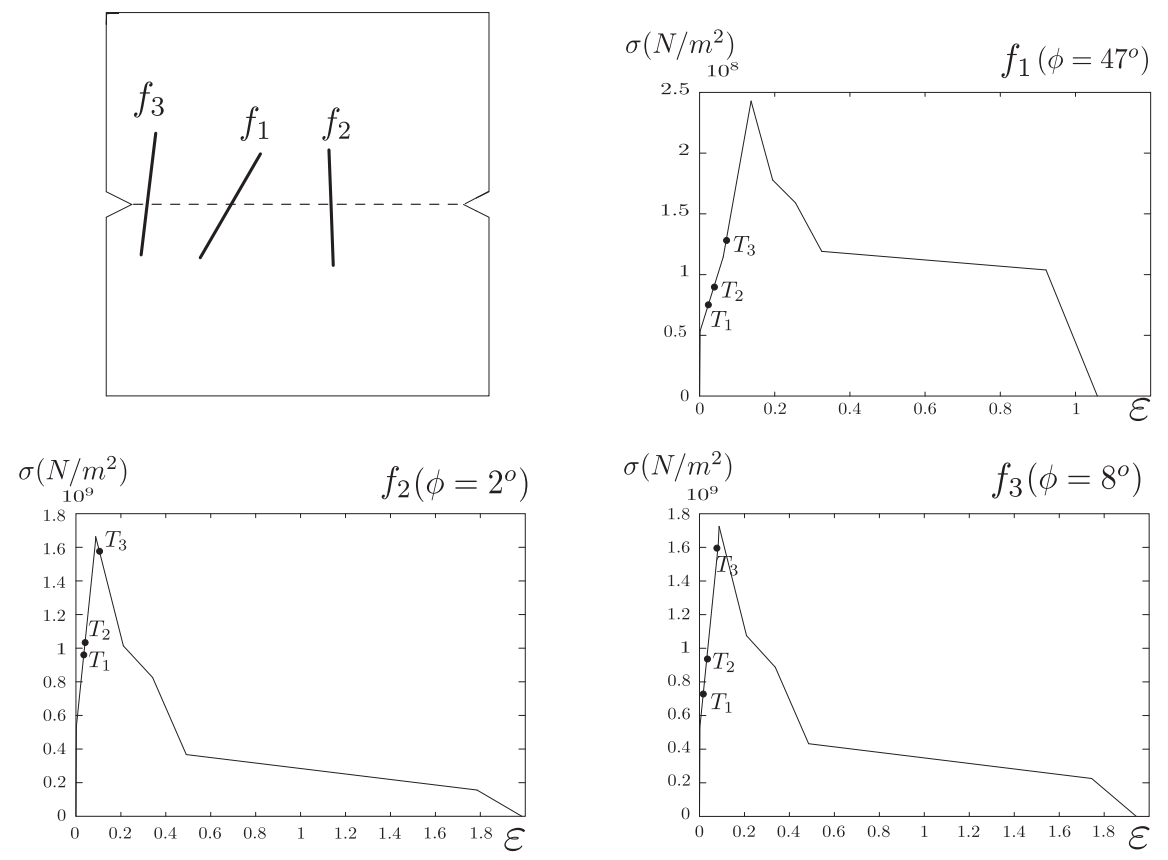

Figure 21. Hooked fibers: behavior of three hooked fibers $\left(f_{1}, f_{2}\right.$, and $\left.f_{3}\right)$ crossing the crack with the constitutive equation of each fiber.

this context, the 3D extension is straightforward and allows more realistic fiber distributions (in 2D, all the fibers are assumed to be coplanar).

In the application examples (two pullout tests with different fiber orientation and the direct tension test), the proposed strategy provides results in qualitative agreement with the experiments and expectations. For instance, the results of the direct tension test of SFRC show that both the energy dissipation and residual strength increase when fibers are added.

\section{APPENDIX A: PARAMETRIZATION OF THE CONSTITUTIVE EQUATIONS FOR STEEL FIBERS DEPENDING ON THE ANGLE}

For a given steel fiber, its constitutive equation is calculated without taking into account the other fibers. The computation of the equations is based on the results from the pullout tests, which are presented in $[14,28,29]$. The analytical expressions deduced from the pullout tests are different for each fiber shape (straight or hooked), therefore, the two different cases are presented independently.

\section{Straight fibers}

For a given steel fiber, once the angle between this fiber and the normal direction of the failure pattern, $\phi$, is computed, the corresponding constitutive equation is calculated. Based on [28], the constitutive equations are defined phenomenologically considering five points $\left(\varepsilon_{i}, \sigma_{i}\right)$, as presented in Figure A.1.

Input data for the phenomenological model include fiber's properties as fiber diameter $(d)$, fiber length $(L)$, and shorter fiber embedded length within the concrete matrix $\left(L_{e}\right)$; concrete properties as the average tensile strength $\left(f_{c t m}\right)$; experimental results of the aligned fiber pullout test $\left(w_{S 01}\right.$, $\left.P_{S 01}\right)$ and $\left(w_{S 02}, P_{S 02}\right)$; numerical parameters as the element size of the fiber $\left(L_{\text {elem }}\right)$ and the area of the fiber $\left(A_{\text {fiber }}\right)$; and finally, the friction coefficient $(\mu)$, and the number of sides of the cracked section at which spalling of the matrix occurs sensitive to fiber orientation $(N)$.

Considering all the input parameters, the five points are defined

(i) $\varepsilon_{1}=\frac{w_{S 01} \cos \phi}{L}$ 


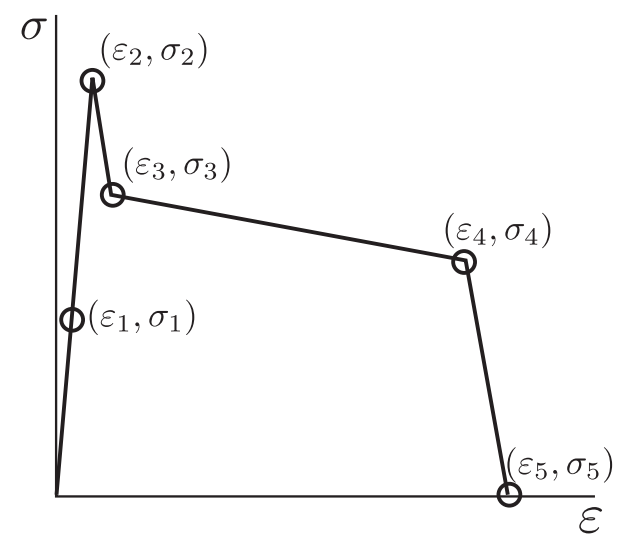

Figure A.1. Phenomenological constitutive equation of a straight fiber defined by five points.

$$
\sigma_{1}=\frac{P_{S 01}}{A_{\text {fiber }}}
$$

(ii) $\varepsilon_{2}=\varepsilon_{1}+\frac{\left(w_{S 01} \cos \phi+\frac{L_{d}}{L_{e}}\left(w_{S 02}-w_{S 01}\right) \cos \phi+\Delta w_{S P 1}\right) \cos \phi}{L_{\text {elem }}}-\frac{w_{S 01} \cos \phi}{L_{\text {elem }}}$

$\sigma_{2}=\frac{P_{S 01} \cos \phi L_{\mathrm{eff}}\left(\frac{L_{e}-L_{d}}{L_{e}}+\frac{P_{S 02} L_{d}}{P_{S 01} L_{e}}\right)+\mu D_{F 1}}{A_{\mathrm{fiber}} \cos \phi}$

(iii) $\varepsilon_{3}=\varepsilon_{1}+\frac{\left(w_{S 02} \cos \phi+\Delta w_{S P 1}\right) \cos \phi}{L_{\text {elem }}}-\frac{w_{S 01} \cos \phi}{L_{\text {elem }}}$

$\sigma_{3}=\frac{P_{S 02} \cos \phi L_{\text {eff }}+\mu D_{F 1}}{A_{\text {fiber }} \cos \phi}$

(iv) $\varepsilon_{4}=\varepsilon_{1}+\frac{\left(L_{e}-\left(L_{S P 1}+d\right)\right) \cos \phi}{L_{\text {elem }}}-\frac{w_{S 01} \cos \phi}{L_{\text {elem }}}$

$\sigma_{4}=\frac{\mu D_{F 1}}{A_{\text {fiber }} \cos \phi}$

(v) $\varepsilon_{5}=\varepsilon_{1}+\frac{\left(L_{e}-L_{S P 1}\right) \cos \phi}{L_{\text {elem }}}-\frac{w_{S 01} \cos \phi}{L_{\text {elem }}}$

$\sigma_{5}=0$

(vi) being

- $L_{S P 1}$, the matrix spalled length which satisfies $a L_{S P 1}^{2}+b L_{S P 1}+c=0$ with $a=\frac{\sqrt{2}}{\sin \phi}+\frac{\cos \phi}{\sin ^{2} \phi}$, $b=\frac{d}{\sin \phi}$ and $c=-\frac{P_{S 01} \sin \phi}{f_{c t m}}$

- $L_{d} \approx 6 L_{S P 1}$ if $6 L_{S P 1} \leqslant L_{e}$. Otherwise, $L_{d}=L_{e}$

- $\Delta w_{S P 1}=N L_{S P 1}(1-\cos \phi)$

- $L_{\text {eff }}=\frac{L_{e}-L_{S P 1}}{L_{e}}$

- $D_{F 1}=P_{S 01} \sin \phi \cos \frac{\phi}{2}$

For the examples studied in the present work, the input parameters used are presented in Table A.1, as introduced in [28].

\section{Hooked fibers}

As in the previous case, for hooked fibers, the constitutive equations are also defined depending on the angle $(\phi)$ and considering different points. However, because of the fiber shape, the interaction is more complex than for the straight fibers. Therefore, eight points $\left(\varepsilon_{i}, \sigma_{i}\right)$ are required for describing the constitutive equations, as shown in Figure A.2, as presented in [29].

Table A.1. Input parameters for straight fibers.

\begin{tabular}{lcccccccr}
\hline$P_{S 01}(\mathrm{~N})$ & $P_{S 02}(\mathrm{~N})$ & $w_{S 01}(\mathrm{~mm})$ & $w_{S 02}(\mathrm{~mm})$ & $d(\mathrm{~mm})$ & $L_{e}(\mathrm{~mm})$ & $f_{c t m}(\mathrm{MPa})$ & $\mu$ & $N$ \\
\hline 44.9 & 12 & 0.05 & 0.4 & 0.5 & 10 & 2.8 & 0.6 & 1 \\
\hline
\end{tabular}




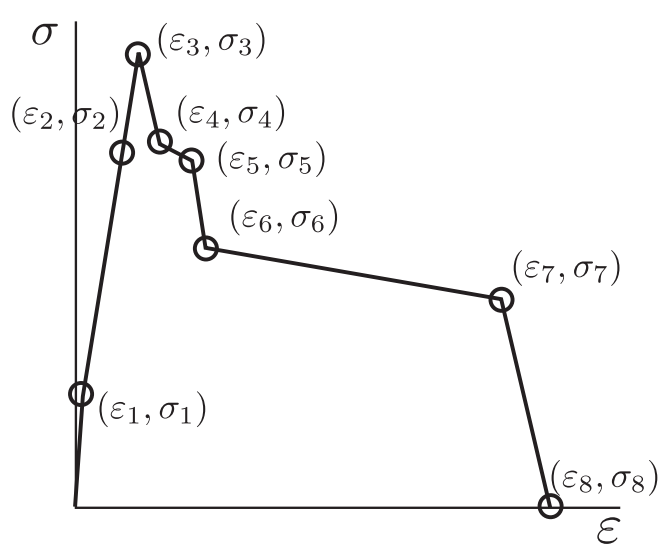

Figure A.2. Phenomenological constitutive equation of a hooked fiber defined by eight points.

Input data for the phenomenological model include fiber's properties as fiber diameter $(d)$, fiber length $(L)$, shorter fiber embedded length within the concrete matrix $\left(L_{e}\right)$, and the ultimate tensile strength of aligned steel fibers $\left(\sigma_{u}\right)$; concrete properties as the average tensile strength $\left(f_{c t m}\right)$; experimental results of the aligned fiber pullout test $\left(w_{S 01}, P_{S 01}\right)$, and $\left(w_{S 02}, P_{S 02}\right)$; experimental information from pullout test derived from the direction of the original embedded part of the fiber $\left(w_{H 01}, P_{H 01}\right),\left(w_{H 02}, P_{H 02}\right),\left(w_{H 03}, P_{H 03}\right)$, and $\left(w_{H 04}, P_{H 04}\right)$; numerical parameters as the element size of the fiber $\left(L_{\text {elem }}\right)$ and the area of the fiber $\left(A_{\text {fiber }}\right)$; and finally, the friction coefficient $(\mu)$, the number of sides of the cracked section at which spalling of the matrix occurs sensitive to fiber orientation $(N)$ and a parameter taking into account the pullout test configuration $(\kappa)$.

Considering all the input parameters, the eight points are defined

$$
\begin{aligned}
& \text { (i) } \varepsilon_{1}=\frac{w_{S 01} \cos \phi}{L} \\
& \sigma_{1}=\frac{P_{S 01}}{A_{\text {fiber }}} \\
& \text { (ii) } \varepsilon_{2}=\varepsilon_{1}+\frac{\left(w_{S 01}+\Delta w_{H 01} \cos \phi \frac{L_{e}-6 \cdot L_{S P 1}}{L_{e}}+\Delta w_{S P 1}\right) \cos \phi}{L_{\mathrm{elem}}}-\frac{w_{S 01} \cos \phi}{L_{\mathrm{elem}}} \\
& \sigma_{2}=\frac{\left(P_{S 01} L_{\mathrm{eff}(H 2)}+\Delta P_{H 01} \frac{L_{e}-6 \cdot L_{S P 1}}{L_{e}}\right) \cos \phi+\mu D_{F 1}}{A_{\mathrm{fiber}} \cos \phi} \\
& \text { (iii) } \varepsilon_{3}=\varepsilon_{1}+\frac{\left(w_{S 01}+\Delta w_{H 01} \cos \phi+\Delta w_{S P 1}+\Delta w_{S P 2}\right) \cos \phi}{L_{\text {elem }}}-\frac{w_{S 01} \cos \phi}{L_{\text {elem }}} \\
& \sigma_{3}=\frac{\left(P_{S 01} L_{\text {eff }(H 3)}+\Delta P_{H 01}\right) \cos \phi+\mu D_{F 2}}{A_{\text {fiber }} \cos \phi} \\
& \text { (iv) } \varepsilon_{4}=\varepsilon_{1}+\varepsilon_{3}+\frac{\Delta w_{H 02} \cos ^{2} \phi}{L_{\text {elem }}}-\frac{w_{S 01} \cos \phi}{L_{\text {elem }}} \\
& \sigma_{4}=\frac{\left(P_{S 01} L_{\mathrm{eff}(H 4)}+\sum_{i=1}^{2} \Delta P_{H 0 i}\right) \cos \phi+\mu D_{F 2}}{A_{\mathrm{fiber}} \cos \phi} \\
& \text { (v) } \varepsilon_{5}=\varepsilon_{1}+\varepsilon_{4}+\frac{\Delta w_{H 03} \cos ^{2} \phi}{L_{\text {elem }}}-\frac{w_{S 01} \cos \phi}{L_{\text {elem }}} \\
& \sigma_{5}=\frac{\left(P_{S 01} L_{\text {eff }(H 5)}+\sum_{i=1}^{3} \Delta P_{H 0 i}\right) \cos \phi+\mu D_{F 2}}{A_{\text {fiber }} \cos \phi} \\
& \text { (vi) } \varepsilon_{6}=\varepsilon_{1}+\varepsilon_{5}+\frac{\Delta w_{H 04} \cos ^{2} \phi}{L_{\text {elem }}}-\frac{w_{S 01} \cos \phi}{L_{\text {elem }}} \\
& \sigma_{6}=\frac{\left(P_{S 02} L_{\mathrm{eff}(H 6)}+\sum_{i=1}^{4} \Delta P_{H 0 i}\right) \cos \phi+\mu D_{F 2}}{A_{\mathrm{fiber}} \cos \phi} \\
& \sigma_{7}=\frac{\left.\sum_{i=1}^{4} \Delta P_{H 0 i}\right) \cos \phi+\mu D_{F 2}}{A_{\text {fiber }} \cos \phi}
\end{aligned}
$$


Table A.2. Input parameters for hooked fibers.

\begin{tabular}{ccccccc}
\hline$P_{S 01}(\mathrm{~N})$ & $P_{S 02}(\mathrm{~N})$ & $w_{S 01}(\mathrm{~mm})$ & $w_{S 02}(\mathrm{~mm})$ & $d(\mathrm{~mm})$ & $L_{e}(\mathrm{~mm})$ & $f_{c t m}(\mathrm{MPa})$ \\
\hline 25 & 12.5 & 0.035 & 0.3 & 0.5 & 20 & 4.46 \\
\hline
\end{tabular}

\begin{tabular}{lccccc}
\hline$\sigma_{u}(\mathrm{MPa})$ & $\mu$ & $N$ & $\kappa$ & $P_{H 01}(\mathrm{~N})$ & $w_{H 01}(\mathrm{~mm})$ \\
\hline 1150 & 0.6 & 1 & 9 & 192 & 0.769 \\
\hline
\end{tabular}

\begin{tabular}{lccccc}
\hline$P_{H 02}(\mathrm{~N})$ & $w_{H 02}(\mathrm{~mm})$ & $P_{H 03}(\mathrm{~N})$ & $w_{H 03}(\mathrm{~mm})$ & $P_{H 04}(\mathrm{~N})$ & $w_{H 04}(\mathrm{~mm})$ \\
\hline 110 & 2.6 & 90 & 4.5 & 65 & 5 \\
\hline
\end{tabular}

(viii) $\varepsilon_{8}=\varepsilon_{1}+\frac{\left(L_{e}-\left(L_{S P 1}+L_{S P 2}\right)\right) \cos \phi}{L_{\text {elem }}}-\frac{w_{S 01} \cos \phi}{L_{\text {elem }}}$

$\sigma_{8}=0$

(ix) being

- $L_{S P 1}$, the matrix spalled length defined for straight fibers

- $L_{S P 2}$ the increment of spalled matrix along fiber axis which satisfies $a L_{S P 2}^{2}+b L_{S P 2}+$ $c=0$ with $a=\frac{\sqrt{2}}{\sin \phi}+\frac{\cos \phi}{\sin ^{2} \phi}, b=\frac{d}{\sin \phi}+\frac{2 L_{S P 1}(\cos \phi+\sqrt{2})}{\sin \phi}$ and $c=\left(-\frac{P_{S 01} \sin \phi}{f_{c t m}}\right) \frac{P_{H 01}}{P_{S 01}}+$ $\frac{L_{S P 1}}{\sin \phi}\left(d+L_{S P 1} \frac{\cos \phi}{\sin \phi}\right)$

- $\Delta P_{H 01}=P_{H 01}-P_{S 01}$ and $\Delta w_{H 01}=w_{H 01}-w_{S 01}$

- for $i=1,2,3, \Delta P_{H 0(i+1)}=P_{H 0(i+1)}-P_{S 0 i}$ and $\Delta w_{H 0(i+1)}=w_{H 0(i+1)}-w_{S 0 i}$

- The effective length factors at each key point $H_{i}$ are defined as

$$
\begin{aligned}
L_{\mathrm{eff}(H 2)} & =\frac{L_{e}-L_{S P 1}}{L_{e}} \\
L_{\mathrm{eff}(H 3)} & =\frac{L_{e}-\left(L_{S P 1}+L_{S P 2}\right)}{L_{e}} \\
L_{\mathrm{eff}(H 4)} & =\frac{L_{e}-\left(L_{S P 1}+L_{S P 2}+\Delta w_{H 02}\right)}{L_{e}} \\
L_{\mathrm{eff}(H 5)} & =\frac{L_{e}-\left(L_{S P 1}+L_{S P 2}+\sum_{i=2}^{3} \Delta w_{H 0 i}\right)}{L_{e}} \\
L_{\mathrm{eff}(H 6)} & =\frac{L_{e}-\left(L_{S P 1}+L_{S P 2}+\sum_{i=2}^{4} \Delta w_{H 0 i}\right)}{L_{e}}
\end{aligned}
$$

- $D_{F 1}=P_{S 01} \sin \phi \cos \frac{\phi}{2}$ and $D_{F 2}=P_{H 01} \sin \phi \cos \frac{\phi}{2}$

- $\Delta w_{S P 1}=N L_{S P 1}(1-\cos \phi)$ and $\Delta w_{S P 2}=L_{S P 2}(1-\cos \phi)$

For the examples studied in the present work, the input parameters used are presented in Table A.2, as introduced in [29].

\section{ACKNOWLEDGEMENTS}

The authors gratefully acknowledge the financial support of the Ministerio de Fomento (Project No. C 59/ 2006) and the Ministerio de Ciencia e Innovación (Project No. BIA2009-13233). 


\section{REFERENCES}

1. EN 14651. Test Method for Metallic Fibred concrete. Measuring the Flexural Tensile Strength (limit of proportionality (LOP), residual). European Committee for Standardization: Brussels, 2005.

2. RILEM TC 162-TDF. Test and design methods for steel fibre reinforced concrete: uniaxial tension test for steel fibre reinforced concrete. Final recommendations. Materials and Structures 2003; 36:560-567.

3. NBN B 15 238. Essais des bétons reforcé de fibres- Essai de flexion sur éprouvettes prismatiques, Institut Belge de Normalisation (IBN), Brussels, 1992.

4. Tschegg EK, Linsbauer HN. Test method for the determination of fracture mechanics properties. Patent specification No. A-233/86 390 328, Austrian Patent Office, 1986.

5. Molins C, Aguado A, Saludes S. Double punch test to control the energy dissipation in tension of FRC (Barcelona test). Materials and Structures 2009; 42(4):415-425.

6. Recommendations of RILEM TC 162-TDF. Test and design methods for steel fibre reinforced concrete Uni-axial tension test for steel fibre reinforced concrete. Materials and Structures 2001; 34(235):3-6.

7. Barragán BE. Failure and toughness of steel fiber reinforced concrete under tension and shear. PhD Thesis, Universitat Politécnica de Catalunya, 2002.

8. Hofstetter G, Peer B, Niederwanger G, Fritsche G. Numerical simulation of ultimate load tests on fiber reinforced concrete beams. VIII International Conference on Computational Plasticity (COMPLAS VIII), Barcelona, Spain, 2005.

9. Zhang J, Li VC. Simulation of crack propagation in fiber-reinforced concrete by fracture mechanics. Cement and Concrete Research 2004; 34:333-339.

10. Schumacher P. Rotation capacity of self-compacting steel fiber reinforced concrete. PhD Thesis, Delft University of Technology, 2006.

11. Markeset G, Hillerborg A. Softening of concrete in compression - localization and size effects. Cement and Concrete Research 1995; 25(4):702-708.

12. Konig G, Kutzing L. Modelling the increase of ductility of HPC under compressive forces - a fracture mechanics approach. High Performance Fiber Reinforced Cement Composites 1999; 6:251-259.

13. Oliver J, Linero DL, Huespe AE, Mansoli OL. Two-dimensional modeling of material failure in reinforced concrete by means of a continuum strong discontinuity approach. Computer Methods in Applied Mechanics and Engineering 2008; 197:332-348.

14. Laranjeira F. Design-oriented constitutive model for steel fiber reinforced concrete. PhD Thesis, Universitat Politècnica de Catalunya, 2010.

15. Phillips DV, Zienkiewicz OC. Finite element non-linear analysis of concrete structures. ICE Proceedings 1976; 61(1):59-88.

16. Balakrishnan S, Murray DW. Finite element prediction of reinforced concrete behavior, 1986. (Available from: www.uofaweb.ualberta.ca/structures/pdfs/SER138BalakrishnanandMurray.pdf.)

17. Elwi AE, Hrudey TM. Finite element model for curved embedded reinforcement. Journal of Engineering Mechanics 1989; 115:740-754.

18. Radtke FKF, Simone A, Sluys LJ. A partition of unity finite element method for obtaining elastic properties of continua with embedded thin fibres. International Journal for Numerical Methods in Engineering 2010; 84(6):708-732.

19. Radtke FKF, Simone A, Sluys LJ. A partition of unity finite element method for simulating non-linear debonding and matrix failure in thin fibre composites. International Journal for Numerical Methods in Engineering 2011; 86(4-5):453-476.

20. Radtke FKF, Simone A, Sluys LJ. A computational model for failure analysis of fibre reinforced concrete with discrete treatment of fibres. Engineering Fracture Mechanics 2010; 77(4):597-620.

21. Pros A, Díez P, Molins C. Numerical modeling of a test assessing the tensile strength of steel fiber reinforced concrete. 8th. World Congress on Computational Mechanics (WCCM8). 5th European Congress on Computational Methods in Applied Sciences and Engineeering (ECCOMAS 2008), Venice, Italy, June 30-July 5, 2008.

22. Boffi D, Gastaldi L. A finite approach of the immersed boundary method. Computers and Structures 2003; 81:491-501.

23. Boffi D, Gastaldi L, Heltai L. On the CFL condition for the finite element immersed boundary method. Computers and Structures 2007; 85:775-783.

24. Mittal R, Iaccarino G. Immersed boundary methods. Annual Review of Fluid Mechanics 2003; 37:239-261.

25. Pros A, Diez P, Molins C. Numerical modeling of double punch test for plain concrete. International Journal of Solids and Structures 2011; 48(7-8):1229-1238.

26. Oliver J, Huespe AE, Cante J C. An implicit/explicit integration scheme to increase computability of nonlinear material and contact/friction problems. Computer Methods in Applied Mechanics and Engineering 2008; 197:1865-1889.

27. Oliver J. Topics on failure mechanics. Monograph CIMNE No. 68, International Center for Numerical Methods in Engineering, 2002.

28. Laranjeira F, Aguado A, Molins C. Predicting the pullout response of inclined straight steel fibers. Materials and Structures 2010; 43:875-895.

29. Laranjeira F, Molins C, Aguado A. Predicting the pullout response of inclined hooked steel fibers. Cement and Concrete Research 2010; 40(10):1471-1487. 